\title{
The metal nanoparticle-induced inflammatory response is regulated by SIRTI through NF-KB deacetylation in aseptic loosening
}

This article was published in the following Dove Press journal:

International Journal of Nanomedicine

10 May 2017

Number of times this article has been viewed

\author{
Zhantao Deng ${ }^{1-3, *}$ \\ Jiewen Jin ${ }^{2,3, *}$ \\ Zhenheng Wang' \\ Yong Wang ${ }^{2,3}$ \\ Qian $\mathrm{Gao}^{2,3}$ \\ Jianning Zhao'
}

'Department of Orthopedics, Jinling Hospital, School of Medicine, Nanjing University, ${ }^{2}$ Center for Translational Medicine, Nanjing University Medical School, ${ }^{3}$ Jiangsu Key Laboratory for Molecular Medicine, Nanjing University Medical School, Nanjing, Jiangsu, People's Republic of China

*These authors contributed equally to this work
Correspondence: Qian Gao

Jiangsu Key Laboratory for Molecular

Medicine, School of Medicine,

Nanjing University, No 22, Hankou

Road, Nanjing 210002, Jiangsu,

People's Republic of China

Email qian_gao@nju.edu.cn

Jianning Zhao

Department of Orthopedics, Jinling Hospital, School of Medicine, Nanjing

University, No 305, Zhongshan

East Road, Nanjing 210002 , Jiangsu,

People's Republic of China

Email zhaojianning.0207@I63.com
Abstract: Aseptic loosening is the most common cause of total hip arthroplasty (THA) failure, and osteolysis induced by wear particles plays a major role in aseptic loosening. Various pathways in multiple cell types contribute to the pathogenesis of osteolysis, but the role of Sirtuin 1 (SIRT1), which can regulate inflammatory responses through its deacetylation, has never been investigated. We hypothesized that the downregulation of SIRT1 in macrophages induced by metal nanoparticles was one of the reasons for osteolysis in THA failure. In this study, the expression of SIRT1 was examined in macrophages stimulated with metal nanoparticles from materials used in prosthetics and in specimens from patients suffering from aseptic loosening. To address whether SIRT1 downregulation triggers these inflammatory responses, the effects of the SIRT1 activator resveratrol on the expression of inflammatory cytokines in metal nanoparticle-stimulated macrophages were tested. The results demonstrated that SIRT1 expression was significantly downregulated in metal nanoparticle-stimulated macrophages and clinical specimens of prosthesis loosening. Pharmacological activation of SIRT1 dramatically reduced the particle-induced expression of inflammatory cytokines in vitro and osteolysis in vivo. Furthermore, SIRT1 regulated particle-induced inflammatory responses through nuclear factor kappa B (NF- $\mathrm{B}$ ) acetylation. Thus, the results of this study suggest that SIRT1 plays a key role in metal nanoparticle-induced inflammatory responses and that targeting the SIRT1 pathway may lead to novel therapeutic approaches for the treatment of aseptic prosthesis loosening.

Keywords: metal nanoparticle, inflammatory response, SIRT1, NF- $\mathrm{B}$, aseptic loosening

\section{Introduction}

Aseptic loosening is the most common cause of total hip arthroplasty (THA) failure, and osteolysis induced by wear particles plays a vital role in its pathological processes. ${ }^{1}$ Among various nanoparticles generated from implant components, metal particles, such as TiAl6V4 particles (TiPs) and CoCrMo particles (CoPs), are among the most common materials to be studied due to the wide use of metal prostheses in clinical practice. ${ }^{2}$ Briefly, nanoparticles generated from implant components stimulate local macrophages and mesenchymal cells to release a multitude of cytokines, chemokines, and metalloproteinases, ${ }^{3,4}$ which subsequently activate osteoclasts and result in bone resorption. ${ }^{5}$ Furthermore, inflammatory cytokines, such as tumor necrosis factor- $\alpha$ (TNF- $\alpha$ ) and interleukin (IL)-1 $\beta$, modulate the balance between osteoblast and osteoclast activities and promote osteolysis. ${ }^{6-9}$ The induction of osteolysis by CoPs could be prevented by the suppression of TNF- $\alpha$ in a murine calvaria osteolysis model. ${ }^{10}$ Endoplasmic reticulum (ER) stress is a mediator in the inflammatory responses induced by both TiPs and CoPs in osteolysis. ${ }^{4}$ However, a specific target for the effective 
regulation of particle-induced inflammatory responses has not been identified, and the precise mechanism by which particles trigger and amplify local inflammatory responses requires further investigation. ${ }^{11-14}$

Macrophages, which secrete various inflammatory mediators/cytokines, are important cells implicated in the initiation of inflammatory responses and play vital roles in the pathogenesis of numerous inflammatory diseases. Nuclear factor kappa B (NF- $\mathrm{KB})$ is a proinflammatory transcription factor and a key mediator of the immune response in macrophages. It consists of five homologous Rel homology domains (RHD), namely, Rel (p65), RelB, CRel (REI), $\mathrm{p} 50$, and p52. ${ }^{15}$ Classic NF- $\mathrm{KB}$ is a dimer of p50 and p65. ${ }^{16,17}$ In an unstimulated state, $\mathrm{NF}-\kappa \mathrm{B}$ is bound to the inhibitory proteins of the inhibitor of $\kappa B$ (I $\kappa B$ ) family by the RHD in the cytoplasm. ${ }^{18,19}$ Once stimulated, NF- $\kappa B$ is liberated and translocates to the nucleus, where it further mediates the transcription of targeted genes, including IL-1 $\beta$ and TNF- $\alpha$.

The mammalian sirtuin family is a group of nicotinamide adenine dinucleotide (NAD+)-dependent deacetylases, including Sirtuin 1 (SIRT1) to SIRT7. Recent studies have revealed that the sirtuin family plays a key role in aging and metabolism, particularly in adapting gene expression and metabolism, according to the cellular energy state. ${ }^{20,21}$ SIRT1, the main member of the sirtuin family and one of the most studied histone deacetylases (HDACs), promotes longevity in many species. In addition to its regulatory roles in metabolism, senescence, and apoptosis, SIRT1 regulates inflammatory responses through the deacetylation of histones in the promoter regions of downstream genes, such as NF- $\mathrm{KB}$ and the activator protein 1 (AP-1). Such deacetylation alters the conformation of chromatin, suppressing the transcriptional activities of transcription factors and ultimately repressing the transcription of inflammation-related genes. ${ }^{19,22}$ Furthermore, clinical evidence suggests that SIRT1 downregulation is closely correlated with inflammatory diseases, while the pharmacological activation of SIRT1 by resveratrol effectively attenuates inflammatory injuries. ${ }^{23-26}$

Therefore, the aims of this study were to examine the effects of metal nanoparticles on SIRT1 and proinflammatory cytokine expression in macrophages and clinical specimens from patients with prosthesis loosening and to investigate the potential role of SIRT1 in inflammatory responses in the pathological process of aseptic loosening.

\section{Materials and methods}

\section{Reagents}

Bovine serum albumin (BSA; A4161), protease inhibitor cocktail (P8340), and resveratrol (R5010) were purchased from Sigma-Aldrich. Fetal bovine serum (FBS; 10099-141) and Dulbecco's Modified Eagle's Medium (DMEM; 11995-065) were obtained from Gibco. RIPA lysis buffer (P0013B) was purchased from Beyotime. EX527 (2780) was purchased from TOCRIS.

\section{Metal nanoparticle preparation}

The TiPs and CoPs were provided by Dr Zhenzhong Zhang from the College of Materials Science and Engineering of Nanjing University of Technology. The characteristics of TiPs and CoPs were observed by transmission electron microscope (TEM). The particle size and distribution of TiPs and CoPs were calculated by SimplePCI software (Compix). To detect the stability of the TiPs and CoPs in the medium, $10 \mathrm{mg}$ of TiPs and CoPs were added to $100 \mathrm{~mL}$ of DMEM and cultured for $24 \mathrm{~h}$. Then, the $\mathrm{pH}$ value in the medium and the mass of particles were detected. For in vitro experiments, the particles were further diluted in cell culture medium to attain concentrations ranging from 10 to $100 \mu \mathrm{g} / \mathrm{mL}$ and ultrasonicated for $20 \mathrm{~min}$ prior to cell exposure.

\section{Cell culture}

The murine macrophage-like cell line Raw264.7, which was obtained from the China Center for Type Culture Collection (Shanghai, China), was cultured in DMEM supplemented with $10 \%$ FBS and $1 \%$ penicillin and streptomycin. The cells were maintained at $37^{\circ} \mathrm{C}$ in a humidified atmosphere of $5 \% \mathrm{CO}_{2}$ and $95 \%$ air.

\section{Patient specimens}

Periprosthetic interface membranes were obtained from four patients undergoing revision operations made necessary by the aseptic loosening of their hip prostheses. Cases in which local infection was present were carefully excluded. The clinical data and X-ray films of these patients are shown in Table 1 and Figure 6A, respectively. A sample obtained from a patient who received an intertrochanteric fracture surgery was used as a control.

\section{Western blotting}

To prepare for Western blotting, the cells, calvarial periosteum of the animals, and periprosthetic interface membranes obtained from patients were first lysed in RIPA lysis buffer (50 mM Tris, $\mathrm{pH}=7.4,150 \mathrm{mM} \mathrm{NaCl}, 1 \%$ sodium deoxycholate, $1 \%$ Triton X-100, $0.1 \%$ sodium dodecyl sulfate [SDS], and $1 \mathrm{mM}$ PMSF) containing a protein inhibitor cocktail for $30 \mathrm{~min}$ on ice and subsequently centrifuged at $12,000 \times g$ for $10 \mathrm{~min}$ at $4^{\circ} \mathrm{C}$. The protein in the supernatants 
Table I Clinical characteristics of patients

\begin{tabular}{llllllll}
\hline Case & Gender & Age (years) & $\begin{array}{l}\text { Years after } \\
\text { implantation }\end{array}$ & $\begin{array}{l}\text { Type of } \\
\text { fixation }\end{array}$ & $\begin{array}{l}\text { Specimen } \\
\text { collection site }\end{array}$ & Diagnosis & $\begin{array}{l}\text { Prosthesis } \\
\text { material }\end{array}$ \\
\hline CON & M & 89 & - & - & Cup & Intertrochanteric fracture & - \\
LOOI & F & 64 & 2 & Cement & Cup & Aseptic loosening & TiAl6V4 \\
LOO2 & F & 48 & 1 & Cement & Cup & Aseptic loosening & TiAl6V4 \\
LOO3 & M & 61 & 1 & Cement & Cup & Aseptic loosening & CoCrMo \\
LOO4 & F & 63 & I & Cement & Cup & Aseptic loosening & TiAl6V4 \\
\hline
\end{tabular}

Notes: Cup, acetabular cup; LOO, aseptic loosening.

Abbreviations: $M$, male; $F$, female.

was collected, and the concentration was determined using a BCA protein assay kit (Beyotime; P0011). Equal amounts of total protein were separated by $10 \%$ sodium dodecyl sulfate-polyacrylamide gel electrophoresis (SDS-PAGE) and subsequently transferred to polyvinylidene fluoride membranes. Western blotting was performed using the following primary antibodies: anti-SIRT1 (Cell Signaling Technology; 2493), anti- $\beta$-actin (Bioworld Technology; AP0733), anti-IкB $\alpha$ (Cell Signaling Technology; 4812), anti-NF- $\kappa$ B p65 (Cell Signaling Technology; C22B4), anti-NF- $\mathrm{KB}$ p65-Acetyl 310 (Abcam; ab19870), antiIRE1 $\alpha$ (Cell Signaling Technology; 3294), and anti-p-JNK
(Cell Signaling Technology; 9251). Subsequently, the following secondary antibodies were applied: horseradish peroxidase-conjugated anti-rabbit IgG (Bioworld Technology; BS13278) and horseradish peroxidase-conjugated anti-mouse IgG (Bioworld Technology; BS12478). After incubation with a specific primary antibody followed by a horseradish peroxidase-conjugated secondary antibody, the protein bands were detected using a chemiluminescence detection system (Syngene, Cambridge, UK). The band density was analyzed using Gene Tools (Syngene). All Western blotting experiments were repeated in triplicate, except for those experiments performed in human tissues.
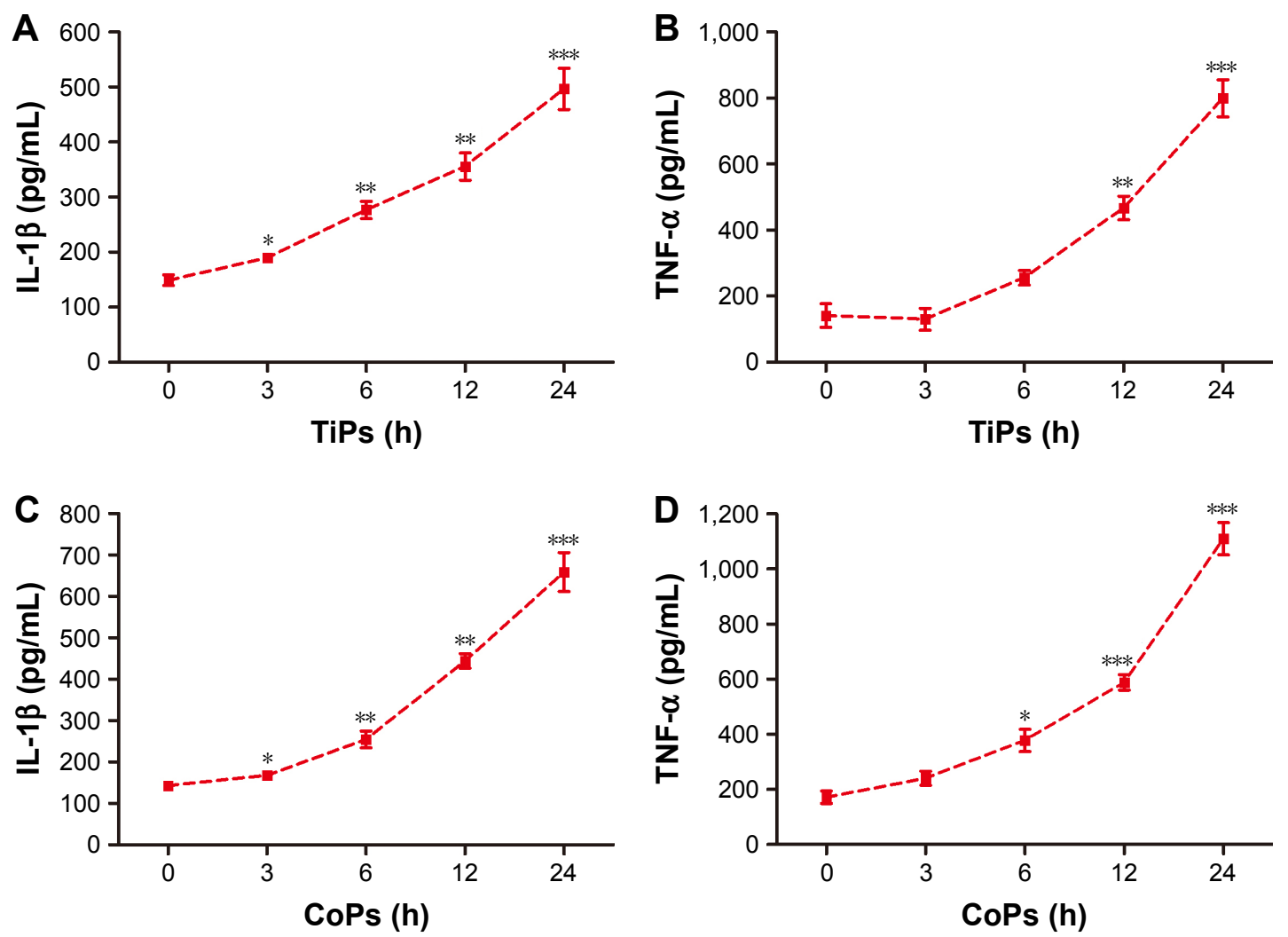

Figure I Metal nanoparticle-induced inflammatory responses in the supernatant of Raw264.7 cells.

Notes: (A-D) The expression of IL-I $\beta$ and TNF- $\alpha$ produced by Raw264.7 cells following incubation with TiPs (I00 $\mu \mathrm{g} / \mathrm{mL})$ or CoPs $(100 \mu \mathrm{g} / \mathrm{mL})$ at the indicated time periods $(0,3,6,12$, or $24 \mathrm{~h})$. $* P<0.05$, $* * P<0.05$, *** $P<0.00 \mathrm{I}$ versus time 0 . The data of all the experiments are represented as the mean \pm SEM from three independent experiments.

Abbreviations: IL, interleukin; TNF, tumor necrosis factor; TiPs, TiAl6V4 particles; CoPs, CoCrMo particles; SEM, standard error of the mean. 


\section{Enzyme-linked immunosorbent assay (ELISA)}

The inflammatory cytokines present in the periosteum and the cell supernatants were quantified using ELISA kits (ExCell Bio; mouse IL-1 $\beta$, EM001, mouse TNF- $\alpha$, EM008, human IL-1 $\beta$, EH001, and human TNF- $\alpha$, EH009). The total protein levels were used as an internal control for the tissue analysis. All procedures were performed in accordance with the manufacturer's instructions.

\section{Immunofluorescence staining}

To detect the SIRT1 expression in Raw264.7 cells and specimens from patients suffering from aseptic loosening, the cells and the slices were stained with an anti-SIRT1 antibody (Cell Signaling Technology; 2493) at $4^{\circ} \mathrm{C}$ overnight. The secondary antibody, Alexa Fluor 647 goat anti-rabbit IgG (Beyotime; P0180), was applied at $37^{\circ} \mathrm{C}$ for $1 \mathrm{~h}$, followed by nuclear staining with 4,6-diamidino-2-phenylindole (DAPI; Beyotime; C1005). The cells and sections were photographed using a Nikon confocal microscope (C2+; Nikon, Tokyo, Japan).

\section{Lentiviral overexpression}

The recombinant lentivirus for SIRT1 overexpression was constructed by Genechem (Shanghai, China), and $1 \times 10^{7}$ transducing unit $(\mathrm{TU}) / \mathrm{mL}$ lentivirus was transfected into Raw264.7 cells for $12 \mathrm{~h}$ before being replaced by fresh media. Subsequently, the cells were cultured for $72 \mathrm{~h}$ before use in further experiments.

\section{Real-time polymerase chain reaction (PCR)}

RNA from the calvarias of the animal models was extracted using the RNA Extraction Kit (Bioteke Co. Ltd; RP5611) following the manufacturer's instructions. The total RNA was reverse transcribed to complementary DNA (cDNA) with PrimeScript ${ }^{\mathrm{TM}}$ RT Master Mix (TaKaRa Co. Ltd; RR036A). SYBR qPCR Mix $(2 \times)$ (TaKaRa Co. Ltd; RR420A) was used for the real-time PCR. All primers are shown in Table 2.

\section{Particle-induced osteolysis (PIO) animal model}

C57BL/J6 mice (6 weeks old) were randomly assigned to the following five groups: group I (mice with the sham operation), group II (TiPs-treated mice), group III (CoPs-treated mice), group IV (TiPs co-treated with resveratrol), and group V (CoPs co-treated with resveratrol). After the mice were anesthetized, we separated the cranial periosteum from the calvarium by sharp dissection and embedded $50 \mu \mathrm{L}$ of metal particle suspensions $(50 \mathrm{mg} / \mathrm{mL})$ into the middle of the calvarias. In the control group, $50 \mu \mathrm{L}$ of phosphate-buffered saline (PBS) instead of metal particle suspensions was applied. In groups IV and V, resveratrol was intragastrically administered at $60 \mathrm{mg} / \mathrm{kg} /$ day for 2 weeks. After 2 weeks, all mice were sacrificed, and the calvarials were harvested for micro-computed tomography (micro-CT) scanning. All the animal experiments were approved by the animal ethics committee of Nanjing University, and all animals received humane care according to the Chinese Laboratory Animal Welfare Requirements and "3R" Principle (Replacement, Reduction, Refinement).

\section{Statistical analysis}

The data are presented as the mean \pm standard error of the mean (SEM). The equality of the group variances was analyzed by the Brown-Forsythe test. The differences among groups were analyzed by one-way analysis of variance (ANOVA). A $P$-value of $<0.05$ indicated a significant difference.

\section{Ethical statement}

The research protocol was approved by the ethics committee of Jingling Hospital, Nanjing, China. Informed consent forms and approval were obtained from all the patients involved in our study. All the animals received humane care according to the Chinese legal requirements.

Table 2 Primer sequences used for real-time RT-PCR

\begin{tabular}{ll}
\hline Gene & Primer sequences forward/reverse \\
\hline GAPDH & $5^{\prime}$-TGTGTCCGTCGTGGATCTGA-3'/5'-TTGCTGTTGAAGTCGCAGGAG-3' \\
iNOS & 5'-CCCTTCCGAAGTTTCTGGCAGCAGC-3'/5'-CCAAAGCCACGAGGCTCTGACAGCC-3' \\
CD86 & $5^{\prime}$-TCAGTCAGGATGGGAGTGGTA-3'/5'-ATCCAAGAGCCATTCCTACCT-3' \\
MR & $5^{\prime}$-CATGAGGCTTCTCTTGCTTCTG-3'/5'-TTGCCGTCTGAACTGAGATGG-3' \\
Arg I & $5^{\prime}$-CAGAAGAATGGAAGAGTCAG-3'/5'-GGTGACTCCCTGCATATCTG-3' \\
\hline
\end{tabular}

Abbreviations: RT-PCR, reverse transcription-polymerase chain reaction; MR, mannose receptor. 


\section{Results}

\section{Cellular SIRTI expression is} downregulated by metal nanoparticles in macrophages

To investigate whether TiPs and CoPs could induce the downregulation of SIRT1 expression in Raw264.7 cells, we analyzed the expression of SIRT1 by Western blot analysis. The Raw264.7 cells were plated at a density of $1 \times 10^{6}$ cells/ well in a 6-well plate and cultured in DMEM for $12 \mathrm{~h}$ prior to being stimulated with TiPs or CoPs at a concentration of $100 \mu \mathrm{g} / \mathrm{mL}$ for various time periods $(0,3,6,12$, or $24 \mathrm{~h})$ or for $24 \mathrm{~h}$ at various concentrations $(0,10,50$, or $100 \mu \mathrm{g} / \mathrm{mL})$. The characteristics of TiPs and CoPs are shown in Figure 2. After stimulation by metal nanoparticles, the SIRT1 protein levels were downregulated in time- and dose-dependent manners (Figure 3). Next, to further confirm the downregulation of cellular SIRT1, we examined SIRT1 expression in Raw264.7 cells in vitro by immunofluorescence staining. Confocal microscopy revealed that the number of cells with SIRT1-positive puncta was obviously decreased after stimulation by metal nanoparticles (Figure 4). Overall, these data indicated that metal nanoparticles induced the downregulation of SIRT1 expression in macrophages.

\section{Metal nanoparticles promote inflammatory responses in macrophages}

To explore the effects of metal nanoparticles on inflammatory cytokine expression in macrophages, Raw264.7 cells were seeded at a density of $1 \times 10^{6}$ cells/well in 6-well plates and exposed to metal particles at a concentration of $100 \mu \mathrm{g} / \mathrm{mL}$ for various time periods $(0,3,6,12$, or $24 \mathrm{~h}$ ). The results showed that both TiPs and CoPs significantly increased IL- $1 \beta$ and TNF- $\alpha$ expression in a time-dependent manner (Figure 1). The maximum induction levels of IL-1 $\beta$ and TNF- $\alpha$ following TiPs treatment were 3.3- and 5.6-fold higher than those observed in the control cells (Figure 1A and $B$ ). The maximum induction of IL- $1 \beta$ and TNF- $\alpha$ in cells treated with CoPs was 4.6- and 6.5-fold higher than that in the control cells (Figure 1C and D).

\section{SIRTI regulates metal nanoparticle- induced inflammatory responses in macrophages}

To further investigate whether the metal nanoparticleinduced inflammatory cytokine upregulation was an outcome of SIRT1 downregulation, Raw264.7 cells were pretreated with $10 \mu \mathrm{M}$ of resveratrol, which activates SIRT1, for $12 \mathrm{~h}$ prior to stimulation with TiPs $(100 \mu \mathrm{g} / \mathrm{mL})$ or CoPs (100 $\mu \mathrm{g} / \mathrm{mL})$ for another $24 \mathrm{~h}$. As shown in Figure 5A-D, the addition of resveratrol downregulated the effects of TiPs and CoPs on cytokine expression. Incubation with resveratrol resulted in a $43 \%$ or $47 \%$ loss, respectively, of IL- $1 \beta$ expression induced by TiPs and CoPs (Figure 5A and $\mathrm{C}$ ), and the expression of TNF- $\alpha$ induced by TiPs and CoPs was reduced by $44 \%$ and $33 \%$, respectively, after treatment with resveratrol (Figure 5B and D). Furthermore, incubation with EX527 (5 $\mu \mathrm{mol} / \mathrm{L})$, a specific SIRT1 inhibitor, reversed the effects of resveratrol on the particle-induced inflammatory responses (Figure 5A-D). To determine whether metal nanoparticles could induce macrophage differentiation, we chose iNOS and CD86 as the biomarkers for M1 macrophages. We chose mannose receptor (MR) and Arg I as the biomarkers for M2 macrophages. The results indicated that metal nanoparticles could induce Raw264.7 cells to differentiate into M1 macrophages, but not M2 macrophages, and could further induce inflammatory cytokine expression. However, resveratrol could only rescue inflammatory responses but could not rescue the phenotype in which Raw264.7 cells differentiated into M1 macrophages (Figure S1).

\section{SIRTI overexpression inhibits inflammatory cytokine production after stimulation by metal nanoparticles}

To further determine if particle-induced inflammatory responses were regulated by SIRT1, a recombinant lentivirus for SIRT1 overexpression was constructed. Similar to the results of pharmacological activation, treatment with metal particles promoted inflammatory responses, and SIRT1 overexpression significantly reduced the levels of particleinduced IL- $1 \beta$ and TNF- $\alpha$ expression. SIRT1 overexpression resulted in a $55.7 \%$ decrease in IL- $1 \beta$ and a $63.0 \%$ decrease in TNF- $\alpha$ with TiPs stimulation, while SIRT1 overexpression resulted in a $48.4 \%$ decrease in IL- $1 \beta$ and a $43.3 \%$ decrease in TNF- $\alpha$ with CoPs stimulation (Figure 5E-H).

\section{SIRTI is downregulated and inflammatory cytokines are overexpressed in clinical specimens of patients with aseptic loosening}

We next performed Western blotting and ELISA analyses using the interface membranes obtained from patients with aseptic loosening. Tissues from a patient with an intertrochanteric 


\section{A}
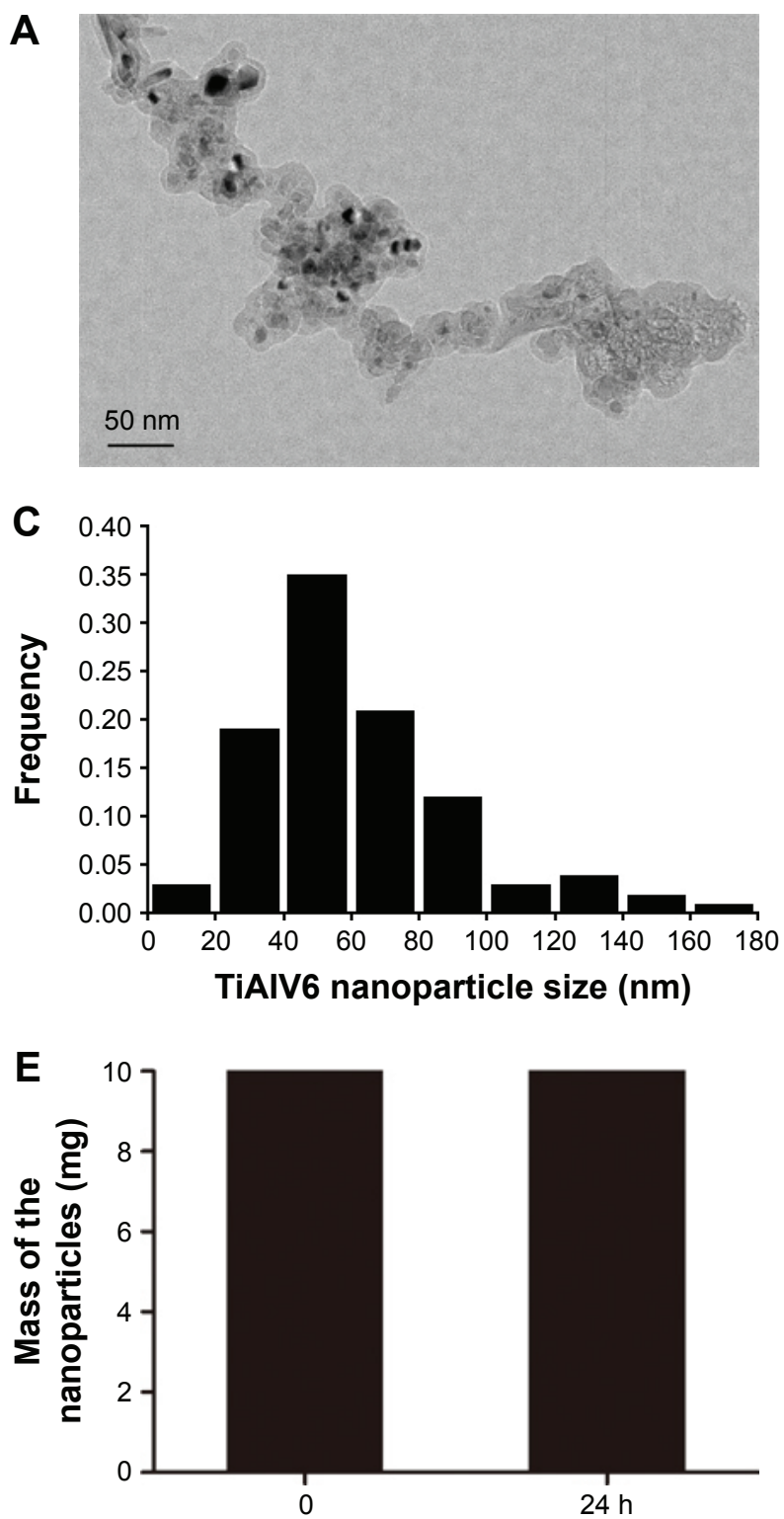

TiAIV6 nanoparticles

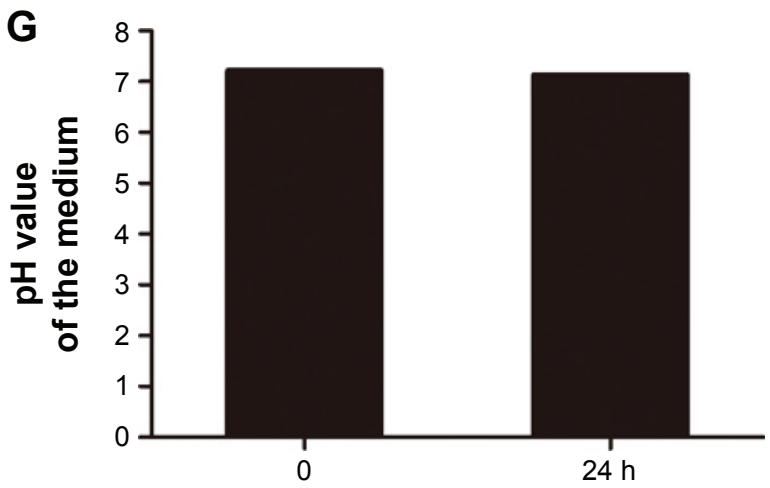

TiAIV6 nanoparticles
B
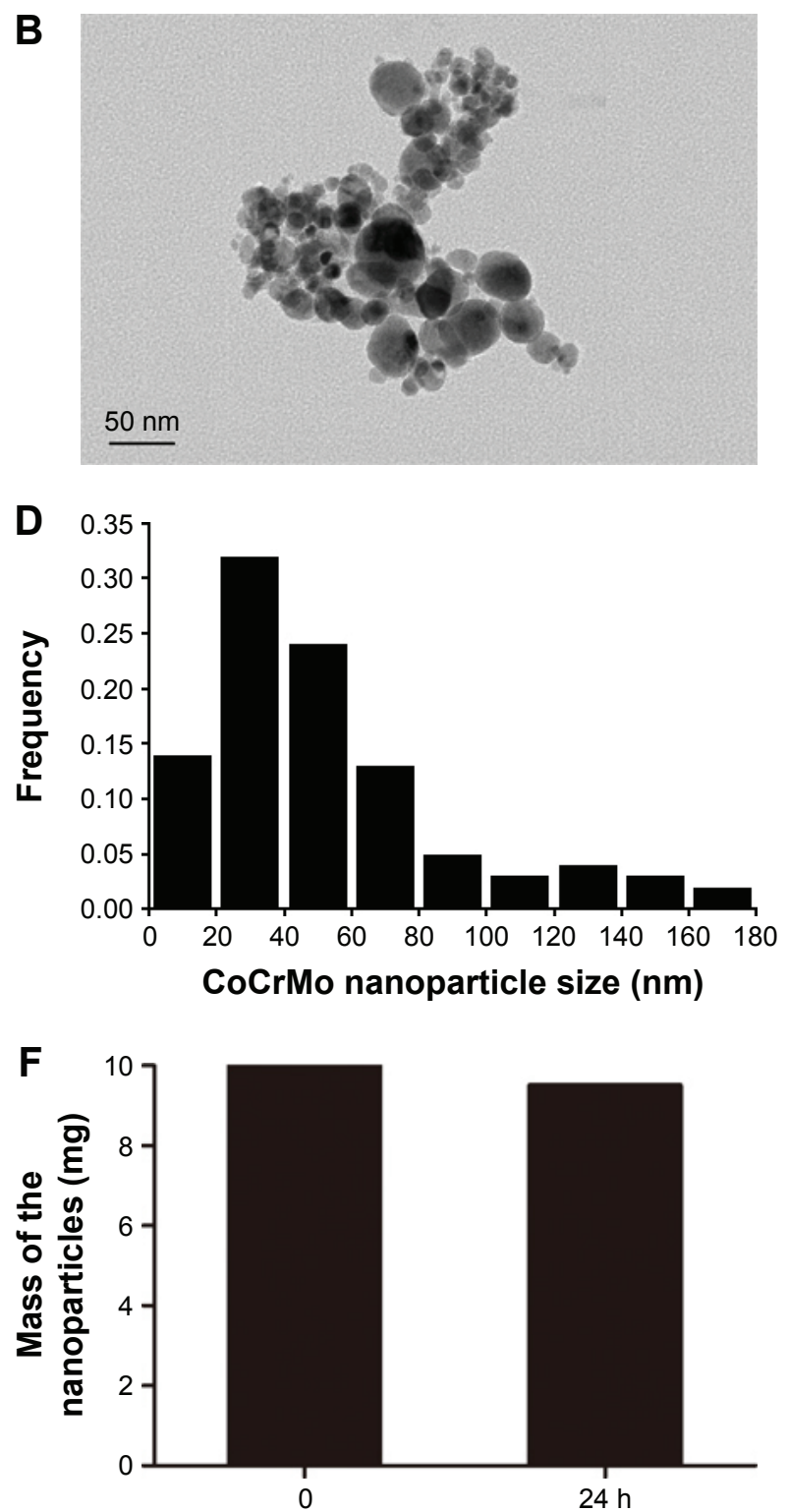

CoCrMo nanoparticles

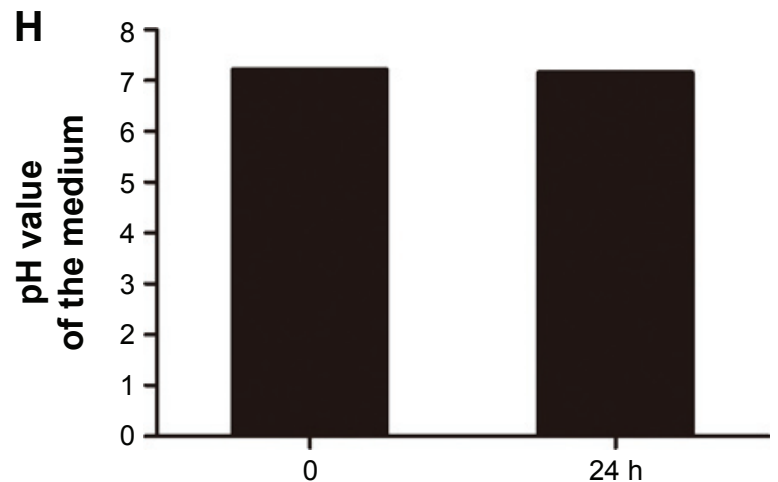

CoCrMo nanoparticles

Figure 2 The characteristics of metal nanoparticles.

Notes: (A, B) TEM images of TiPs and CoPs. (C, D) The particle size distribution of TiPs and CoPs was calculated by SimplePCl software (Compix). (E, F) A total of 10 mg of TiPs and CoPs were cultured in $100 \mathrm{~mL}$ of DMEM, and the mass of the particles was detected after $24 \mathrm{~h}$. (G, $\mathbf{H}$ ) A total of I0 mg of TiPs and CoPs were cultured in I00 mL of DMEM, and the $\mathrm{pH}$ value of the medium was detected.

Abbreviations: TEM, transmission electron microscope; TiPs, TiAl6V4 particles; CoPs, CoCrMo particles; DMEM, Dulbecco's Modified Eagle’s Medium. 
A TiPs concentration $(\mu \mathrm{g} / \mathrm{mL})$

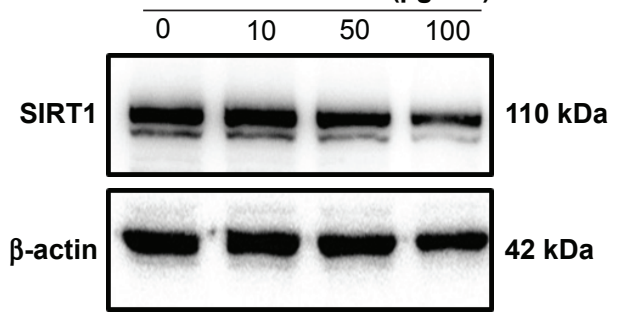

B

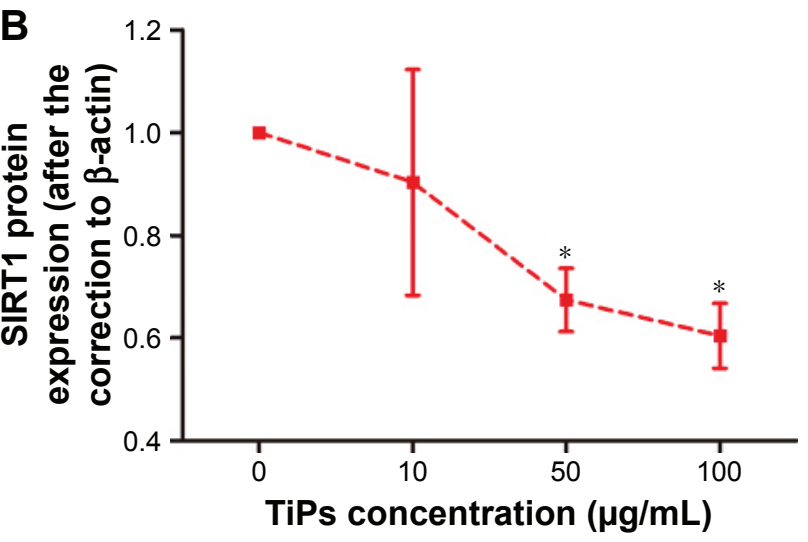

$\mathbf{E}$

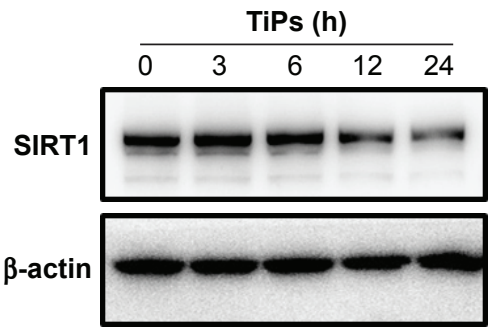

\section{$\mathbf{F}$}

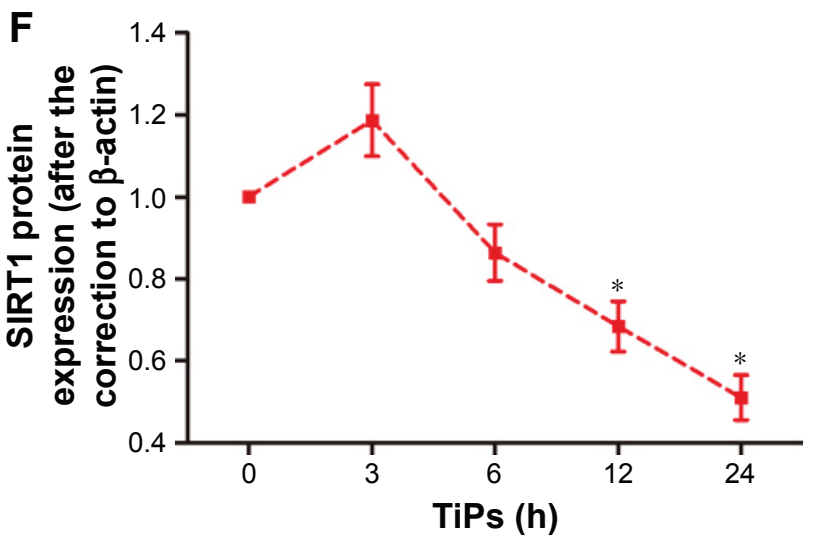

C CoPs concentration $(\mu \mathrm{g} / \mathrm{mL})$
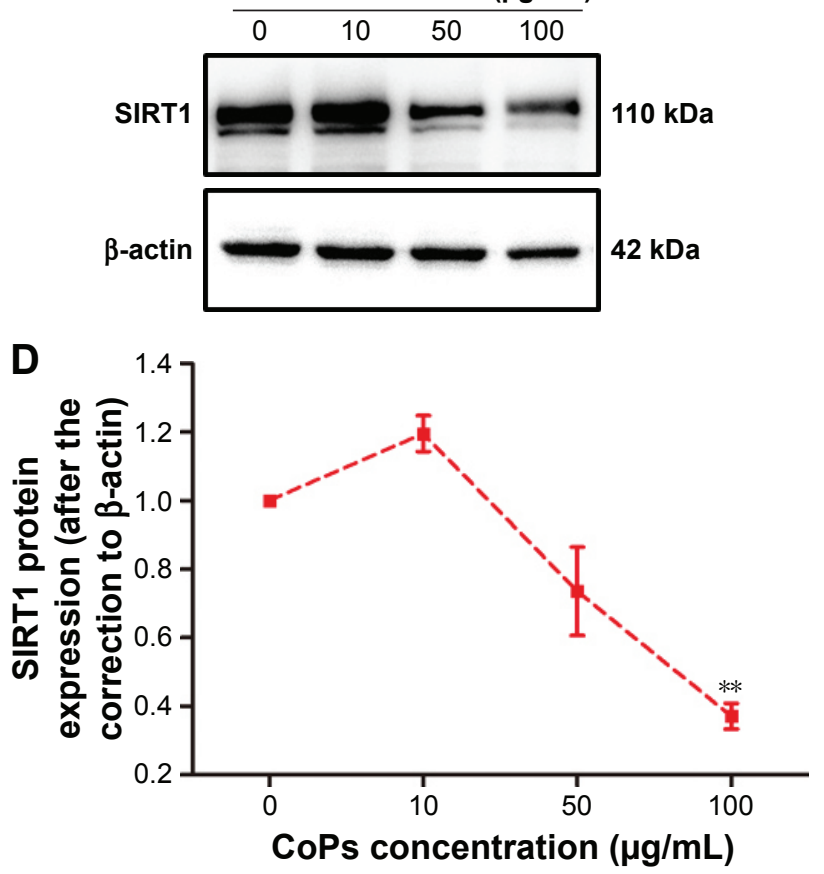

G
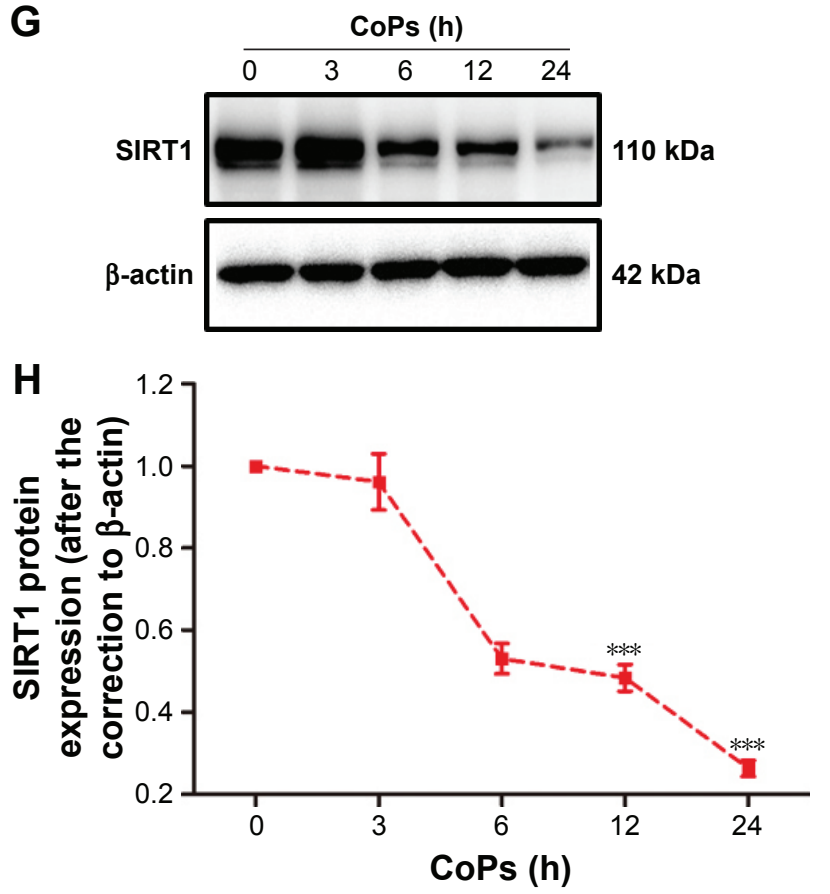

Figure 3 SIRTI expression was downregulated after exposure to metal nanoparticles.

Notes: (A, C) SIRTI protein levels after Raw264.7 cells were incubated with TiPs or CoPs at various concentrations (0, 10, 50, or 100 $\mu \mathrm{g} / \mathrm{mL})$. (B, D) The densities of the Western blot bands in $(\mathbf{A})$ and $(\mathbf{C})$ were quantified using the Gene Tools software. $* P<0.05, * * P<0.01$ versus concentration 0 . (E, G) SIRTI protein levels after Raw264.7 cells were incubated with $100 \mu \mathrm{g} / \mathrm{mL}$ TiPs or CoPs for the indicated time periods $(0,3,6,12$, or $24 \mathrm{~h})$. $(\mathbf{F}, \mathbf{H})$ The density of the Western blot bands in $(\mathbf{E})$ and (G) were quantified using the Gene Tools software. $* P<0.05, * * * P<0.001$ versus time 0 . The data of all the experiments are represented as the mean \pm SEM from three independent experiments.

Abbreviations: SIRTI, Sirtuin I; TiPs, TiAl6V4 particles; CoPs, CoCrMo particles; SEM, standard error of the mean.

fracture were used as a control. The clinical characteristics of the patients are shown in Table 2 and Figure 6A. As shown in Figure 6B, the expression levels of SIRT1 in patients with aseptic loosening were significantly downregulated compared with those of the control. Consistent with the Western blot findings, immunofluorescence staining demonstrated that the expression of SIRT1 was dramatically downregulated at the interface membrane of patients with 

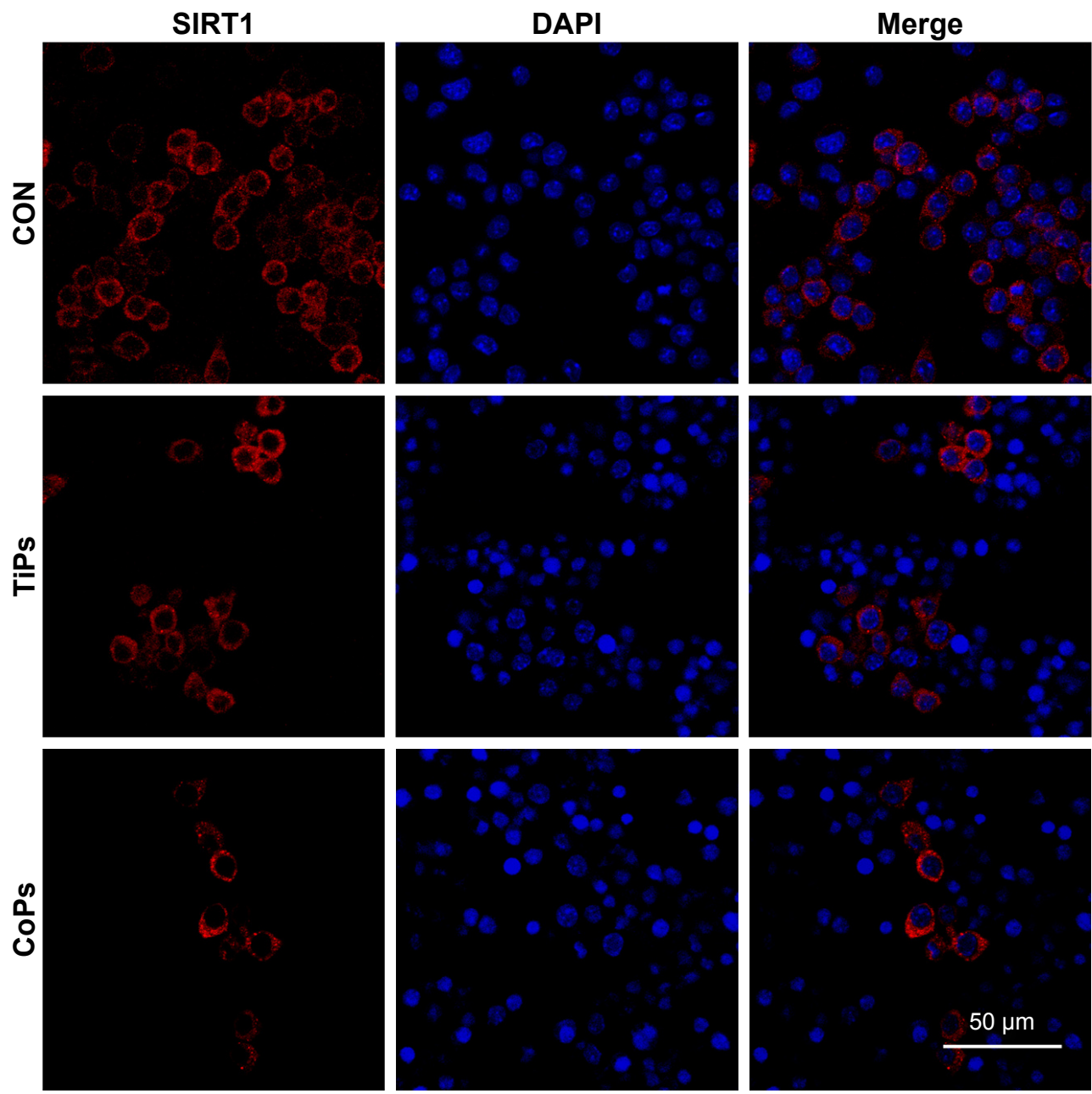

Figure 4 Immunofluorescence was performed to examine the expression of SIRTI in the RAW264.7 cells.

Notes: The cells were treated with $100 \mu \mathrm{g} / \mathrm{mL}$ TiPs or CoPs for $24 \mathrm{~h}$. Red, SIRTI; blue, DAPI nuclear staining.

Abbreviations: SIRTI, Sirtuin I; TiPs, TiAI6V4 particles; CoPs, CoCrMo particles; DAPI, 4,6-diamidino-2-phenylindole; CON, control.

aseptic loosening (Figure 7). Furthermore, we observed the expression of IL- $1 \beta$ and TNF- $\alpha$ in specimens in patients with aseptic loosening. Consistent with the in vitro experiments, the IL- $1 \beta$ and TNF- $\alpha$ levels detected in the interface membranes of patients with aseptic loosening were much higher than those observed in the control group (Figure 6C and D). These results suggested a strong association between SIRT1 expression and aseptic loosening in humans.

\section{The SIRTI-NF-KB pathway is involved in the inflammatory responses induced by metal particles}

Previous studies have suggested that inflammatory responses can be triggered by the SIRT1-NF- $\mathrm{KB}$ signaling pathway. ${ }^{19,27,28}$ Thus, we determined whether the SIRT1$\mathrm{NF}-\kappa \mathrm{B}$ signaling pathway is involved in metal nanoparticleinduced inflammatory responses in macrophages. Both TiPs and CoPs significantly increased the expression of acetylated
NF- $\kappa \mathrm{B}$ (K310) and decreased the expression of I $\kappa \mathrm{B} \alpha$ in vivo and in vitro. However, no obvious differences in the total NF- $\kappa \mathrm{B}$ expression were observed among the different groups (Figures 6B and 8). Furthermore, we detected the suppression of the NF- $\mathrm{KB}$ pathway using particles and resveratrol co-treatment. We observed significantly decreased levels of acetylated NF- $\kappa \mathrm{B}$ and increased levels of $\mathrm{I} \kappa \mathrm{B} \alpha$, but not total NF- $\kappa \mathrm{B}$, after treatment with resveratrol. These effects could be reversed by treatment with EX527 (Figure 8A-D). Next, to confirm whether particle-induced inflammatory responses were mediated by the NF- $\mathrm{KB}$ signaling pathway, Raw264.7 cells were co-treated with metal nanoparticles and BAY-11-7082, an NF- $\kappa$ B inhibitor. As shown in Figure 9, TNF- $\alpha$ and IL-1 $\beta$ expression levels were significantly reduced in cells treated with BAY-11-7082. Collectively, these results indicated that the observed particle-induced inflammatory responses were mediated by the SIRT1-NF-кB pathway. 

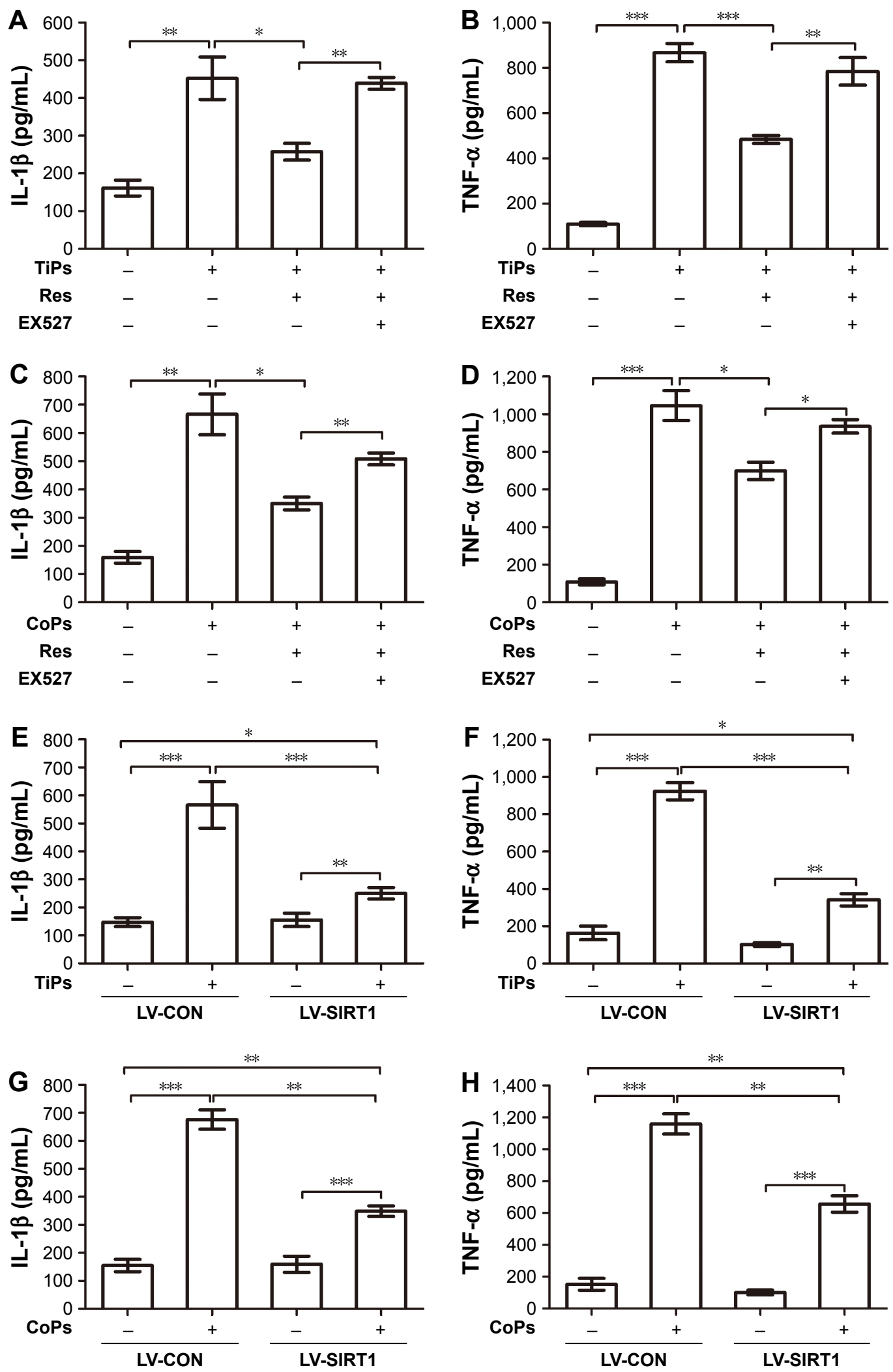

Figure 5 SIRTI regulates the induction of inflammatory cytokine expression by metal nanoparticles in the supernatant of Raw264.7 cells.

Notes: (A-D) Inhibition of the expression of inflammatory cytokines by resveratrol. The expression of inflammatory cytokines in Raw 264.7 cells incubated with resveratrol $(10 \mu \mathrm{M})$ for $12 \mathrm{~h}$ prior to stimulation with TiPs $(100 \mu \mathrm{g} / \mathrm{mL})$ or CoPs $(100 \mu \mathrm{g} / \mathrm{mL})$ for another $24 \mathrm{~h}$. EX527 (5 $\mu \mathrm{M})$ was used to reverse the effects of resveratrol. $* P<0.05$, $* * P<0.05$, ***P $<0.00$ I. (E-H) Inhibition of the expression of inflammatory cytokines by SIRTI overexpression. Control cells and SIRTI-overexpressing cells were stimulated with TiPs $(100 \mu \mathrm{g} / \mathrm{mL})$ or CoPs $(100 \mu \mathrm{g} / \mathrm{mL})$ for $24 \mathrm{~h}$. $* P<0.05$, $* * P<0.05$, $* * * P<0.001$. The data of all the experiments are represented as the mean \pm SEM from three independent experiments.

Abbreviations: SIRTI, Sirtuin I; TiPs, TiAI6V4 particles; CoPs, CoCrMo particles; SEM, standard error of the mean; Res, resveratrol; LV-CON, lentivirus control. 
A

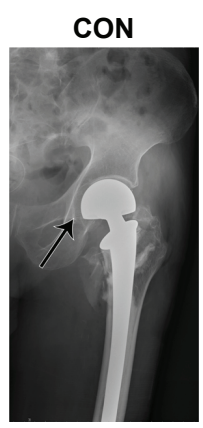

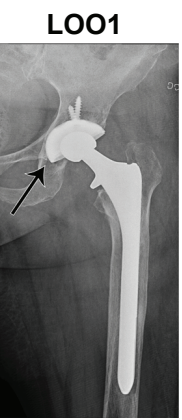
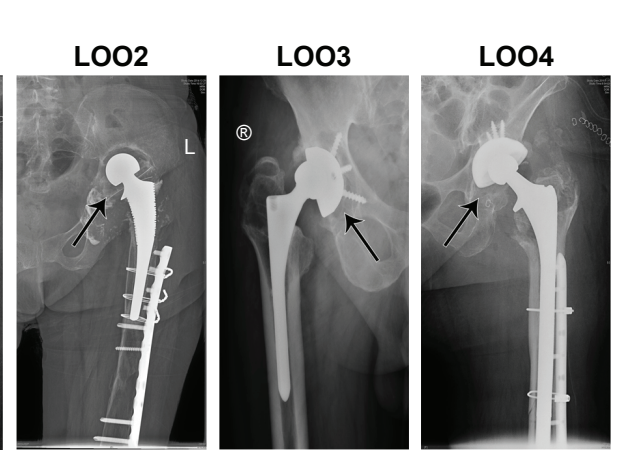

B

B

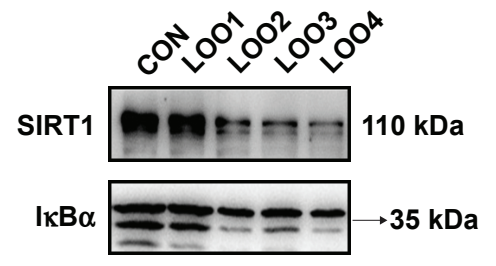

NF-kB p65-
Acetyl $310 \square \mathrm{kDa}$
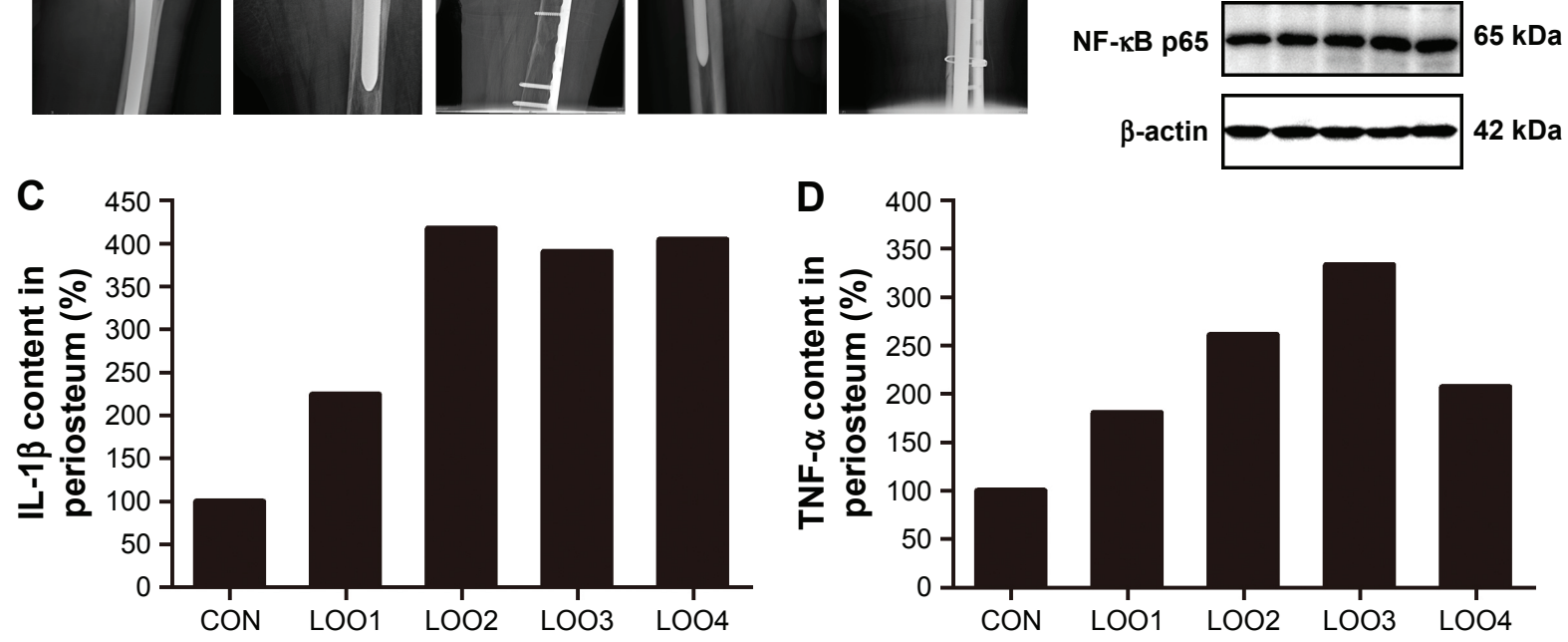

Figure 6 SIRTI was associated with the induction of inflammatory cytokine expression by metal particles in clinical specimens of aseptic loosening.

Notes: (A) Radiographic films of patients with aseptic loosening. The arrow indicates the site where the specimens were obtained. (B) Western blots of clinical specimens of aseptic loosening. (C, D) The expression of inflammatory cytokines of clinical specimens of aseptic loosening. The cytokine concentrations were quantified by ELISA. Abbreviations: SIRTI, Sirtuin I; ELISA, enzyme-linked immunosorbent assay; LOO, aseptic loosening; I $\kappa B$, inhibitor of $\kappa B$; NF- $\kappa B$, nuclear factor kappa B; IL, interleukin; TNF, tumor necrosis factor.

\section{SIRTI activation attenuates osteolysis and local inflammatory responses in PIO animal models}

PIO animal models were constructed by TiPs and CoPs. Not only was the expression of SIRT1 in the periosteum downregulated compared to that in the sham operation group, but the NF- $\mathrm{KB}$ signaling was also activated (Figure 10A and B). Micro-CT with three-dimensional reconstruction was applied to reveal osteolysis induced by metal nanoparticle implantation. Figure 10C demonstrates that PIO in PIO animal models was suppressed by the administration of resveratrol. On the other hand, the expression of TNF- $\alpha$ and IL- $1 \beta$ in the periosteum of PIO animal models was also increased by metal particle implantation, and this effect could be rescued by resveratrol (Figure 10D and E). All the results in the PIO animal models were consistent with the results of the in vitro experiments.

\section{Discussion}

THA is one of the most successful surgical procedures to be developed in recent decades; however, the ratio of revision of arthroplasty remains relatively high because of osteolysis induced by metal nanoparticles and the subsequent aseptic loosening..$^{29,30}$ The metal nanoparticles are predominately phagocytosed by resident macrophages, which secrete various inflammatory cytokines and chemokines, such as TNF- $\alpha$ and IL-1 $\beta$. The local inflammatory response makes a vital contribution to the pathogenesis of osteolysis, and macrophages are the predominant cells involved in the inflammatory response. The presence of inflammatory cytokines such as TNF- $\alpha$ and IL- $1 \beta$ at the interface tissue creates an environment suitable for osteoclast differentiation and osteolysis. ${ }^{31}$ In addition, TNF- $\alpha$ directly activates osteoclasts and upregulates the expression of the receptor activator of NF- $\mathrm{KB}$ ligand (RANKL) in osteoblastic cells, ${ }^{32}$ leading to the recruitment of RANK-positive monocytes/ macrophages, the differentiation of macrophage precursors into giant cells or osteoclasts, and the stimulation of mature osteoclasts to resorb bone. ${ }^{14,32,33}$ In this study, both TiPs and CoPs induced macrophages to differentiate into M1 macrophages and promoted the secretion of TNF- $\alpha$ and IL-1 $\beta$. Based on previous studies concerning inflammatory cytokines and bone metabolism and the observation that both TiPs and CoPs were able to stimulate the production of 

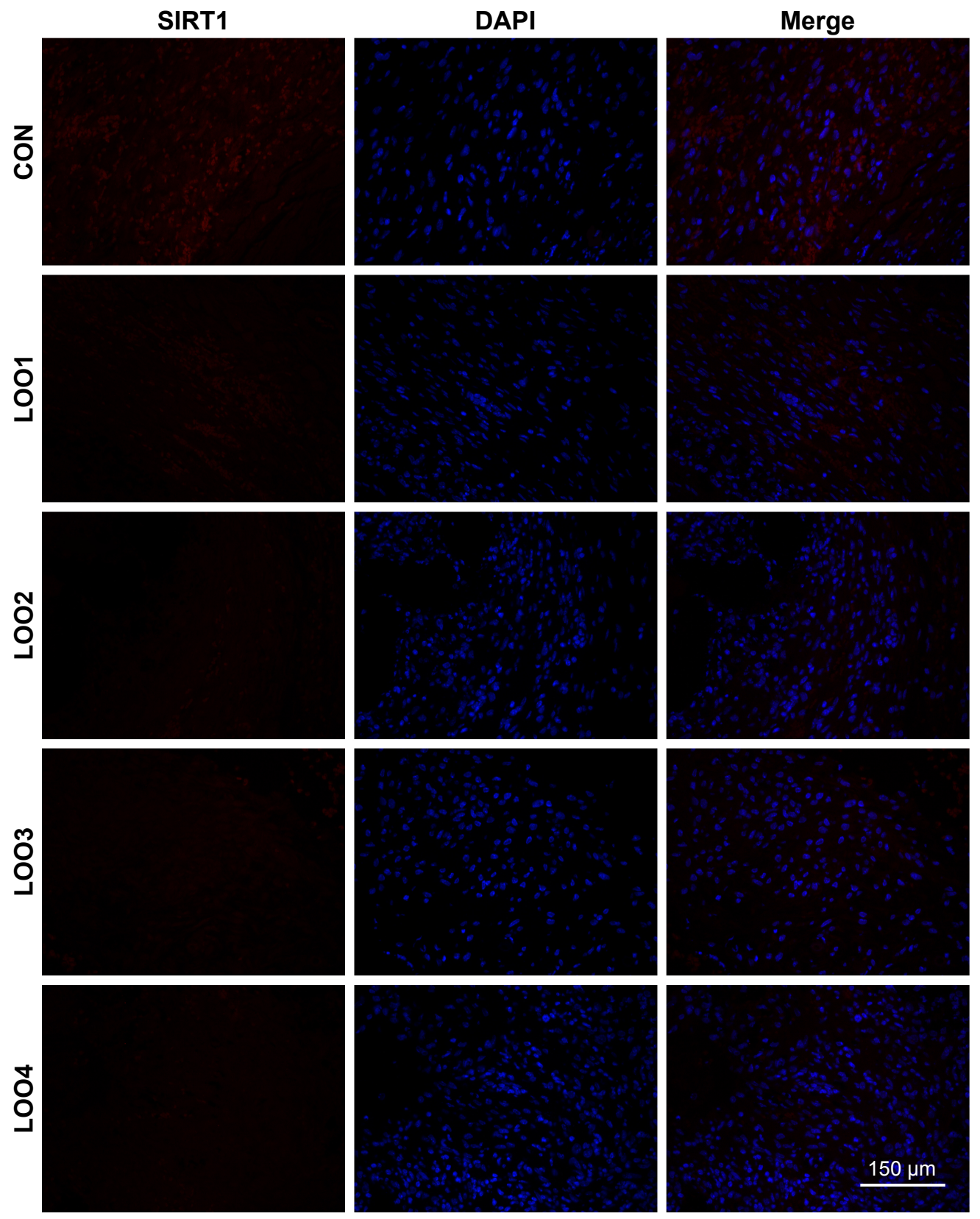

Figure 7 SIRTI was downregulated in the clinical specimens of patients with aseptic loosening.

Notes: Immunofluorescence was performed to examine the expression of SIRTI in the clinical specimens of patients with aseptic loosening. Red, SIRTI; blue, DAPI nuclear staining.

Abbreviations: SIRTI, Sirtuin I; DAPI, 4,6-diamidino-2-phenylindole; LOO, aseptic loosening; CON, control.

TNF- $\alpha$ and IL- $1 \beta$ in this study, it was reasonable to speculate that TNF- $\alpha$ and IL-1 $\beta$, produced by macrophages after the stimulation of metal nanoparticles, mediated the balance between bone resorption and formation in the pathological process of aseptic loosening (Figure S2). 4,34-39

SIRT1 is an important HDAC due to its proposed role in the association between calorie restriction and lifespan extension. SIRT1 is also a major regulator of bone mass. SIRT1 represses the expression of sclerostin (SOST) by deacetylating $\mathrm{H} 3 \mathrm{~K} 9$ at its promoter region. In female mice with SIRT1 haploinsufficiency, the SOST levels were substantially increased, and bone formation and bone mass were reduced. ${ }^{40}$ Furthermore, SIRT1 exerts its multiple activities by interacting with not only histones but also numerous enzymes, transcription factors, and other protein species. ${ }^{41}$ For example, SIRT1 was able to activate runt-related transcription factor 2 (RUNX2) and suppress NF- $\mathrm{\kappa B}$ signaling in bone, leading to the stimulation of osteoblastogenesis and the inhibition of osteoclastogenesis. ${ }^{42-45}$ In this study, the expression of SIRT1 was decreased in macrophages treated 
with both TiPs and CoPs. After SIRT1 was overexpressed in murine macrophages by using a lentivirus, significant protective effects were observed in the nanoparticle-induced inflammatory responses. Compared with other options for SIRT1 overexpression, such as adenoviruses, retroviruses, or plasmids, lentiviruses can be transfected into macrophages with higher efficiency, can integrate the SIRT1 gene into osteoblasts, and can maintain SIRT1 overexpression in the cell passage cultures. Furthermore, by using an in vivo calvaria resorption model, we demonstrated that SIRT1 expression was associated with PIO, suggesting that SIRT1 is also a bone mass regulator in the pathogenesis of aseptic loosening. Moreover, the particle-induced SIRT1 downregulation promoted inflammatory responses both in vivo and in vitro, suggesting an inseparable relationship among SIRT1 expression, inflammatory responses, and osteolysis.

As scavengers, macrophages, after ingesting nanoparticles such as the TiPs and CoPs, activate the cellular machinery associated with intracellular degradation, such as lysosomes and peroxisomes, ${ }^{46-48}$ increase the levels of reactive oxidants and other free radicals, and initiate ER stress. ${ }^{49-51}$ In our previous study, we demonstrated that ER stress, induced by TiPs
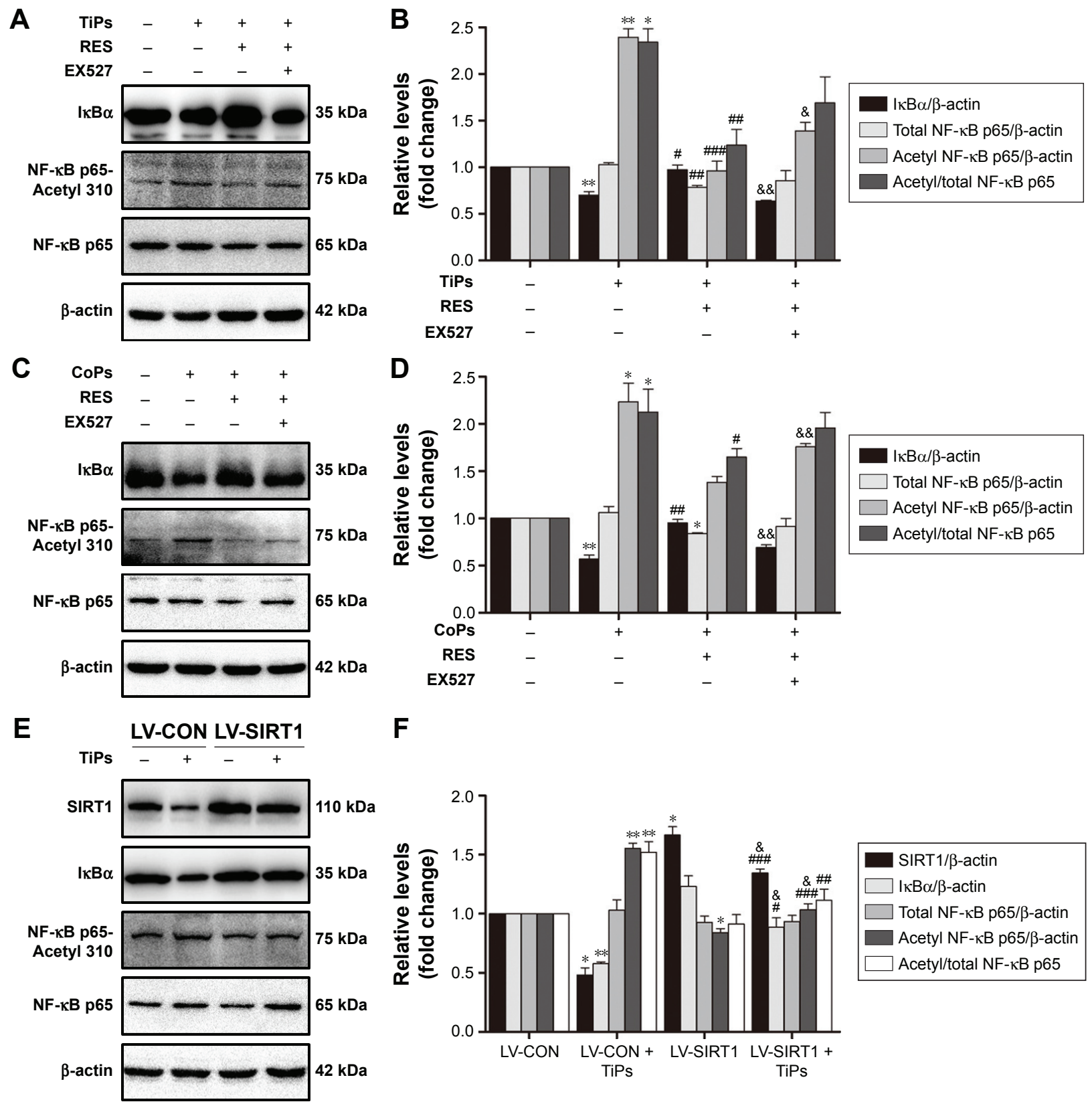

\section{F}

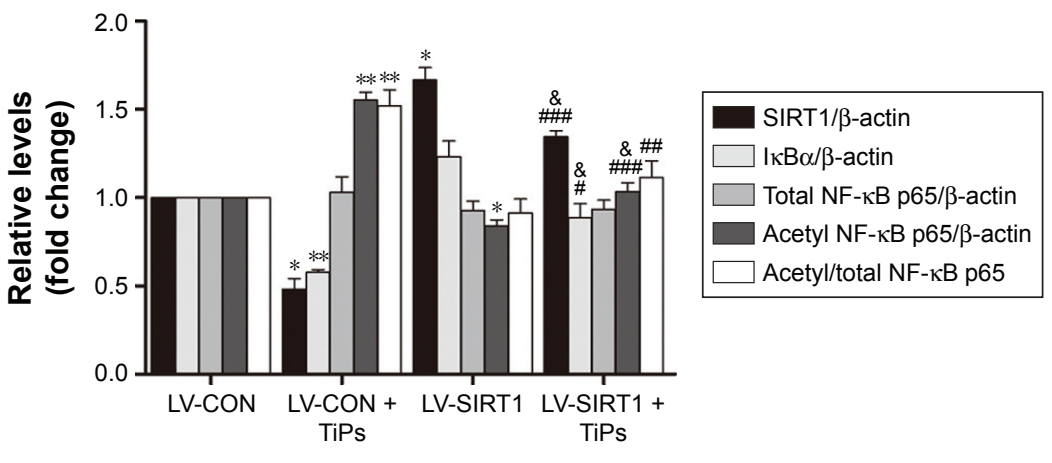

Figure 8 (Continued) 


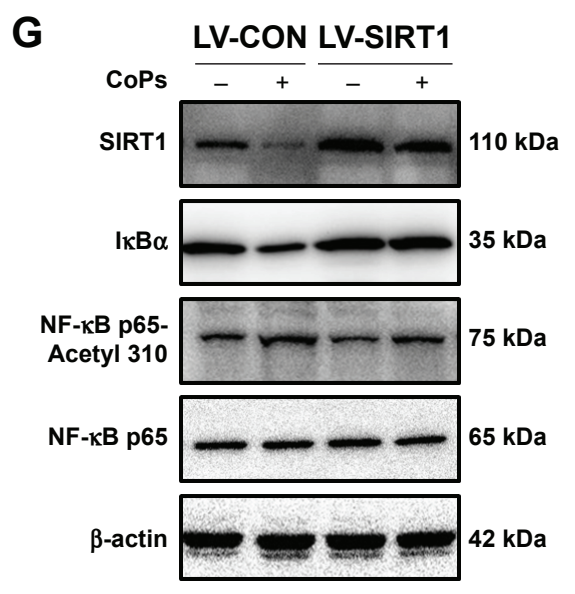

\section{H}

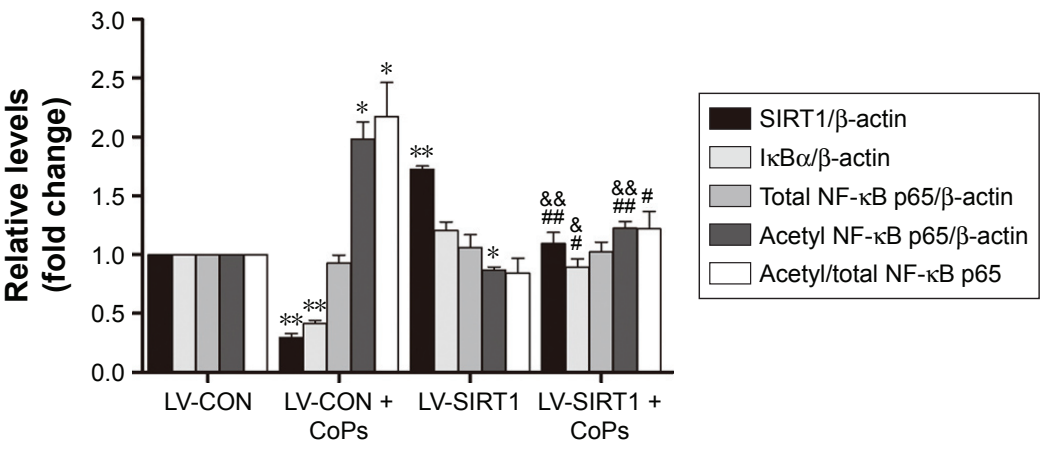

Figure 8 The SIRTI-NF- $\mathrm{KB}$ pathway was involved in the inflammation responses induced by metal nanoparticles.

Notes: (A) Western blots performed after Raw264.7 cells were treated with PBS (control), TiPs, TiPs + resveratrol, TiPs + resveratrol + EX527. (B) The density of the

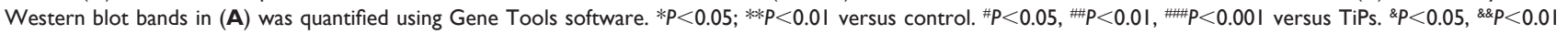
versus TiPs + resveratrol. (C) Western blots performed after Raw264.7 cells were treated with PBS (control), CoPs, CoPs + resveratrol, CoPs + resveratrol + EX527. (D) The density of the Western blot bands in $(\mathbf{C})$ was quantified using the Gene Tools software. ${ }^{*} P<0.05 ; * * P<0.01$ versus control. ${ }^{*} P<0.05$, ${ }^{*} P<0.0$ I versus CoPs. ${ }^{\text {\& }} P<0.01$ versus CoPs + resveratrol. (E) Western blots performed after control cells and SIRTI overexpression cells were treated with TiPs. (F) The density of the Western blot

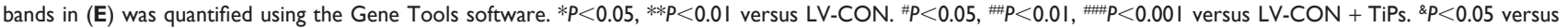
LV-SIRTI. (G) Western blots performed after control cells and SIRTI overexpression cells were treated with CoPs. $(\mathbf{H})$ The density of the Western blot bands in $(\mathbf{G})$ was

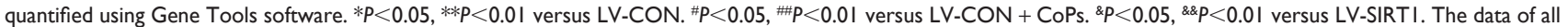
the experiments are represented as the mean \pm SEM from three independent experiments.

Abbreviations: SIRTI, Sirtuin I; NF-אB, nuclear factor kappa B; PBS, phosphate-buffered saline; TiPs, TiAI6V4 particles; CoPs, CoCrMo particles; SEM, standard error of the mean; IKB, inhibitor of $\kappa B$; RES, resveratrol.

A

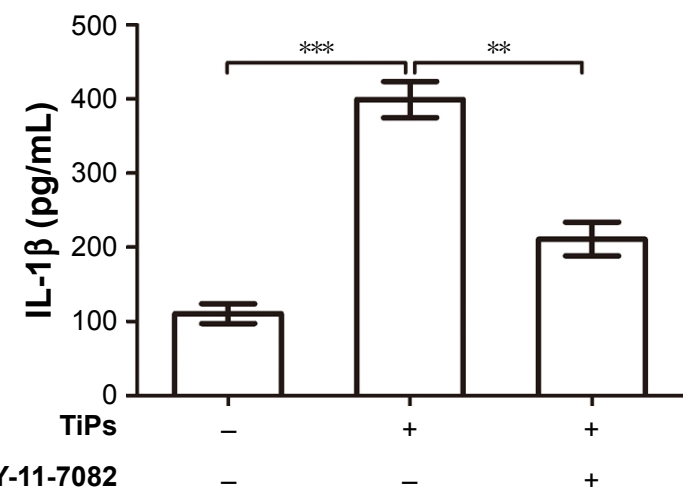

C

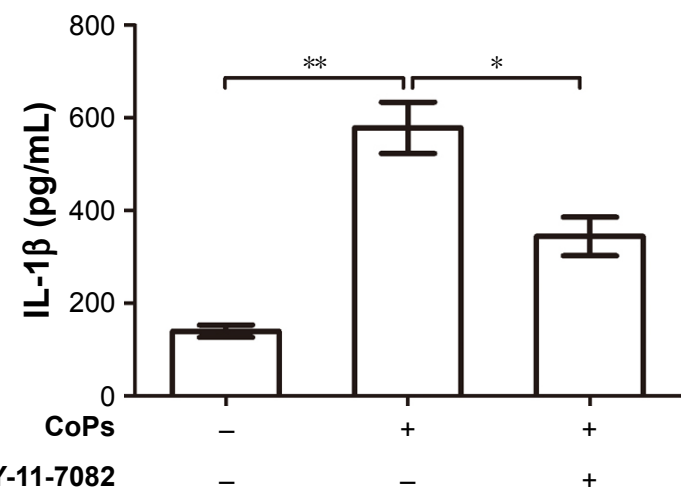

B

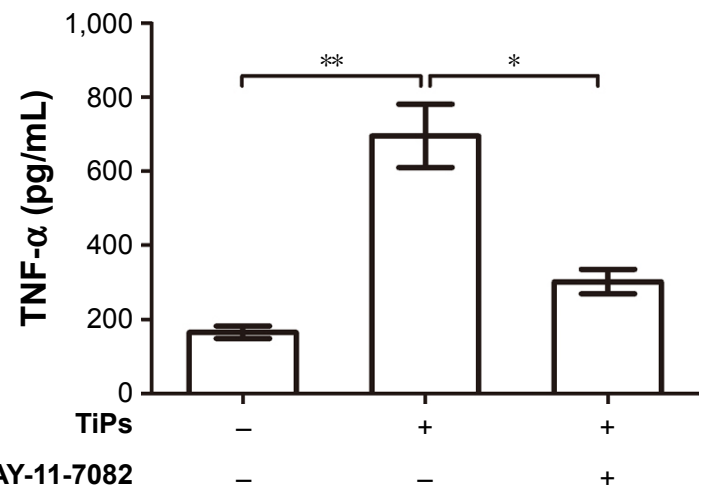

D

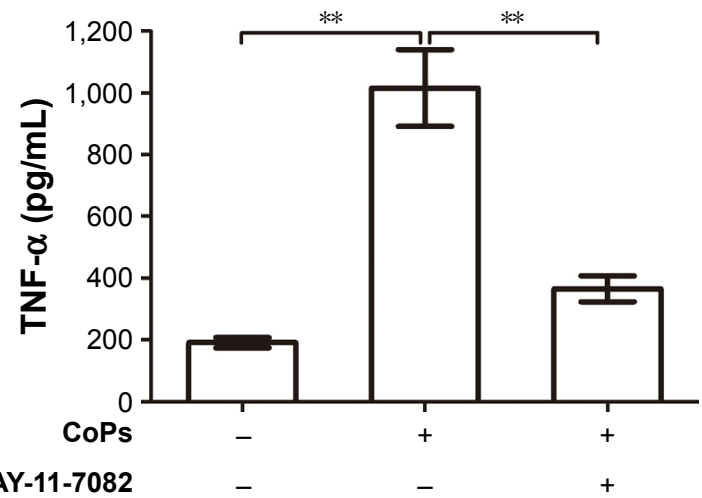

Figure 9 NF-KB inhibitor suppresses metal nanoparticle-induced inflammatory responses in macrophages.

Notes: (A-D) The expression of inflammatory cytokines in the supernatant of Raw264.7 cells after pretreatment with BAY-I I-7082 (I0 $\mu$ M) for $8 \mathrm{~h}$ prior to stimulation with TiPs $(100 \mu \mathrm{g} / \mathrm{mL})$ or CoPs $(100 \mu \mathrm{g} / \mathrm{mL})$ for another $24 \mathrm{~h}$. The cytokine concentrations were quantified by ELISA. $* P<0.05$, $* * P<0.0 \mathrm{I}, * * * P<0.00 \mathrm{I}$. The data of all the experiments are represented as the mean \pm SEM from three independent experiments.

Abbreviations: NF- $\mathrm{KB}$, nuclear factor kappa B; TiPs, TiAl6V4 particles; CoPs, CoCrMo particles; SEM, standard error of the mean; ELISA, enzyme-linked immunosorbent assay; IL, interleukin; TNF, tumor necrosis factor. 
A

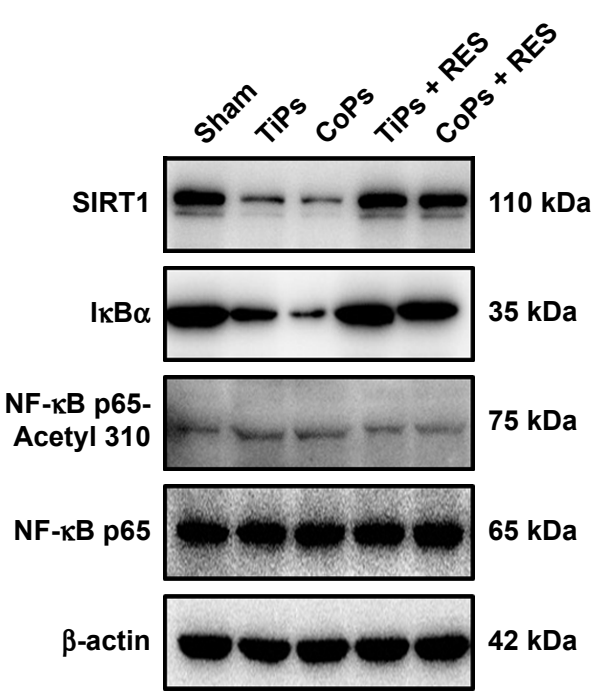

C
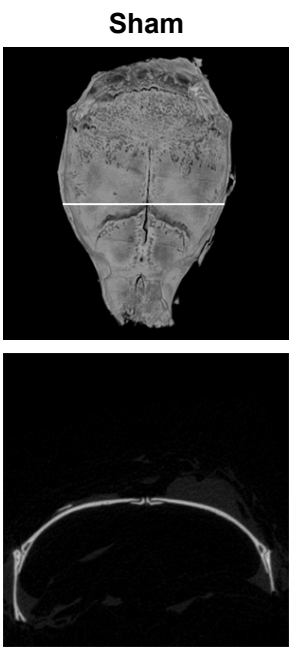

D
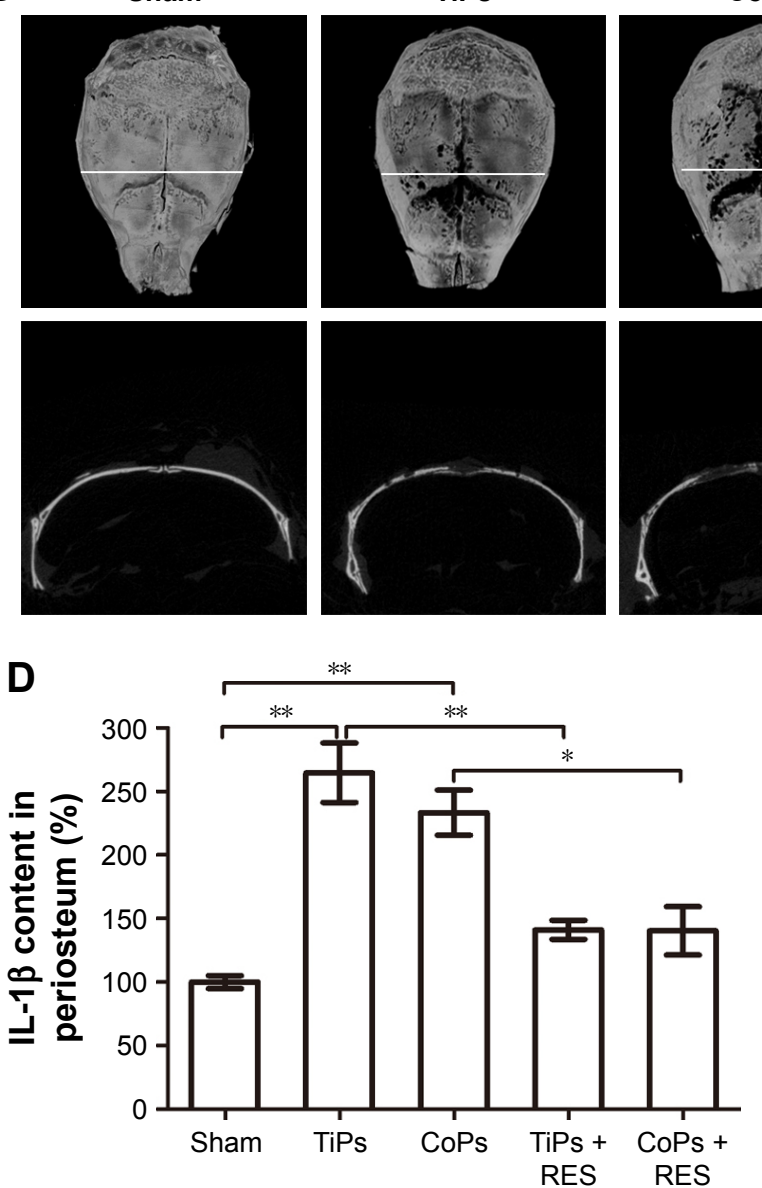
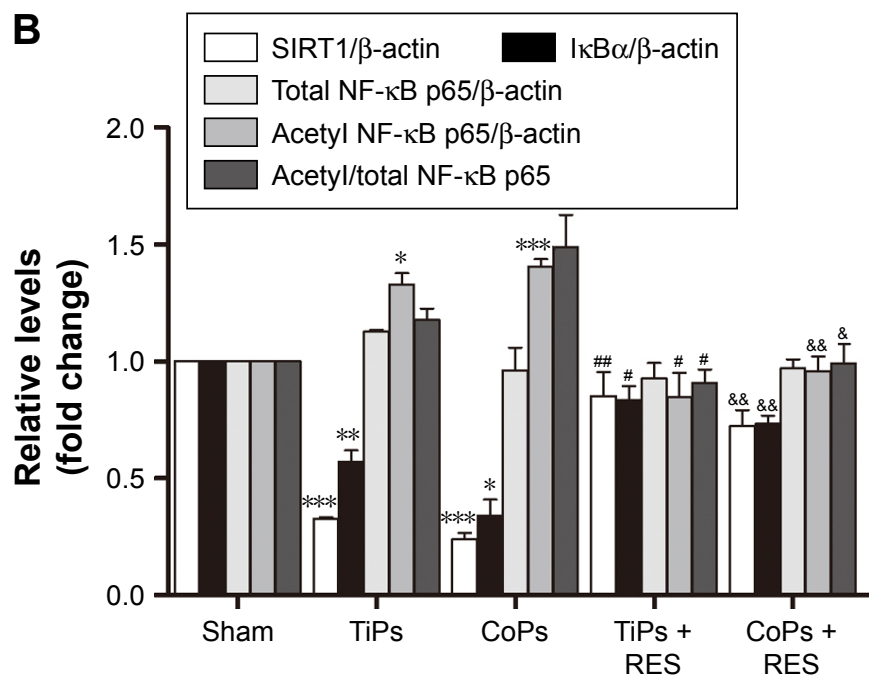

CoPs

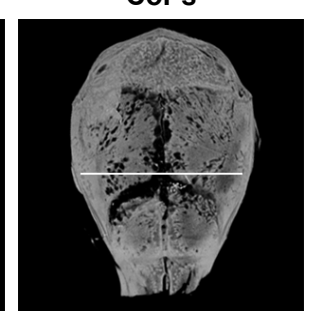

TiPs + RES

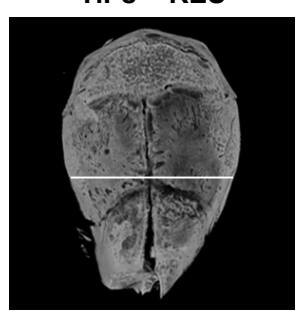

CoPs + RES
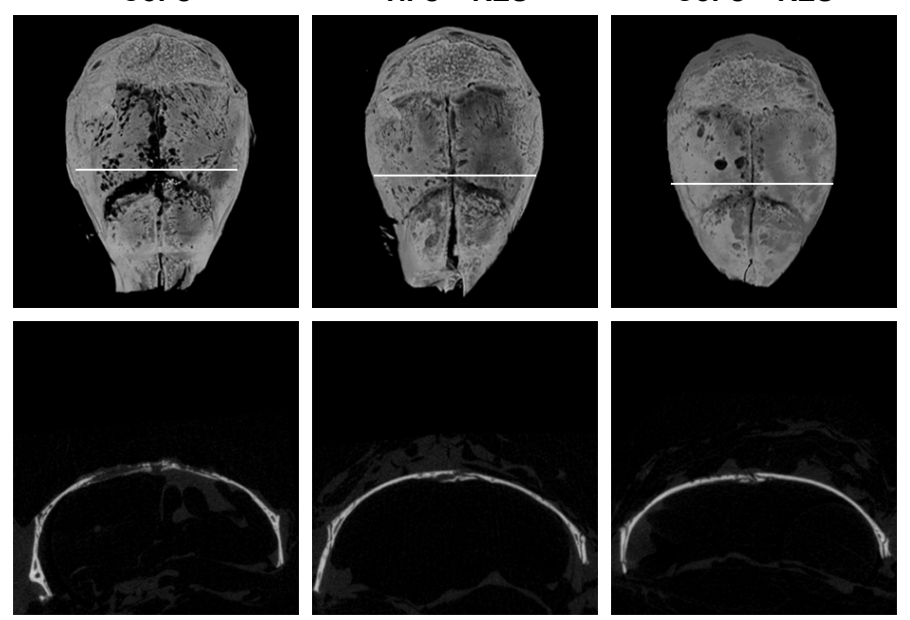

E

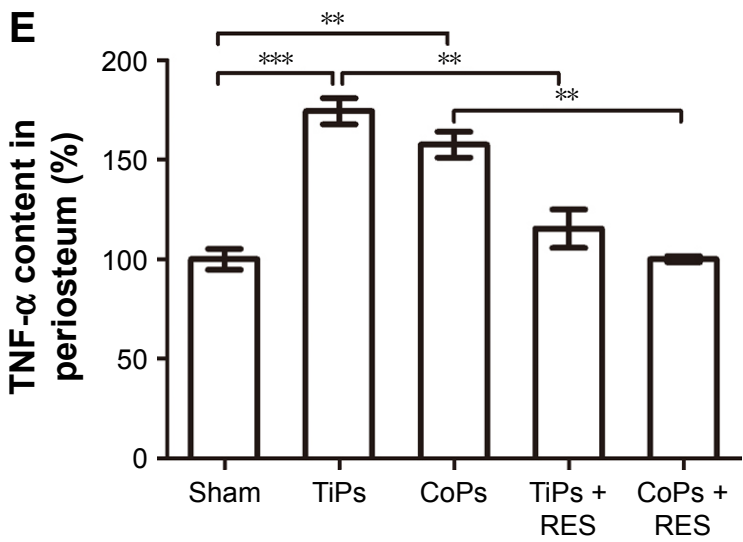

Figure IO SIRTI mediated PIO and local inflammatory responses in animal models.

Notes: (A) Western blots performed in the periosteum of animals treated with PBS (sham), TiPs, CoPs, TiPs + resveratrol, or CoPs + resveratrol. (B) The density of the

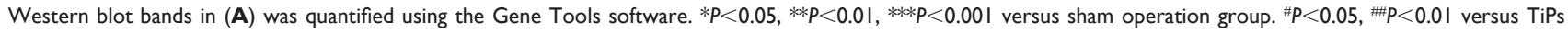
group. ${ }^{\&} P<0.05,{ }^{\& \&} P<0.01$ versus CoPs group. (C) Representative images of micro-CT with 3-dimensional reconstructed images (top panel) from animals treated with PBS (sham), TiPs, CoPs, TiPs + resveratrol, or CoPs + resveratrol. In the second panel, the white horizontal line indicates cross-sectional views of the reconstructed images. (D, E) The levels of TNF- $\alpha$ and IL-I $\beta$ were detected by ELISA in the periosteal tissues of animals treated with PBS (sham), TiPs, CoPs, TiPs + resveratrol, or CoPs + resveratrol. $* P<0.05, * * P<0.01$, $* * * P<0.001$. Data are represented as the mean $\pm S E M$. $n=6$ mice per group.

Abbreviations: SIRTI, Sirtuin I; PIO, particle-induced osteolysis; PBS, phosphate-buffered saline; TiPs, TiAI6V4 particles; CoPs, CoCrMo particles; CT, computed tomography; TNF, tumor necrosis factor; IL, interleukin; ELISA, enzyme-linked immunosorbent assay; SEM, standard error of the mean; IKB, inhibitor of $\kappa B$; NF- $\kappa B$, nuclear factor kappa B; RES, resveratrol. 
and CoPs, mediated the expression of inflammatory cytokines in cultured macrophages and animal models. Using an ER stress inhibitor, the inflammatory response and osteolysis could be ameliorated both in vitro and in vivo. ${ }^{4}$ However, the precise mechanisms underlying the induction of ER stress by TiPs and CoPs in macrophages remain unclear. Interestingly, multiple correlations between SIRT1 and ER stress have been reported (Figure S3A). The activation of SIRT1 by resveratrol, NAD+, or calorie restriction could attenuate ER stress via the PERK/eIF2 $\alpha$, ATF6/CHOP, or IRE1 $\alpha$ / JNK-mediated pathways. ${ }^{52-56}$ In this study, we observed the downregulation of SIRT1 in macrophages treated with both TiPs and CoPs, and the treatment of resveratrol showed a similar effect on the inflammatory response with an ER stress inhibitor. Those results suggest a correlation between ER stress and SIRT1 in the pathological process of aseptic loosening. We also observed that resveratrol could regulate particle-induced ER stress through the IRE1 $\alpha /$ JNK pathway in macrophages, but more evidence is still needed to confirm the relationship between SIRT1 and ER stress in aseptic loosening (Figure S3B).

In recent studies, the treatments for PIO were focused on the inhibition of inflammation and the suppression of osteoclasts. ${ }^{38,57}$ However, these strategies were ineffective in patients and may lead to adverse effects on other organs. The inhibition of TNF may inhibit responses to harmful agents, including bacteria and other infective agents. Bisphosphonate, an inhibitor of osteoclast-mediated bone resorption, may result in the impairment of fracture healing, mandibular lesions, pathologic femoral fractures, and other adverse events. ${ }^{57}$ Thus, new strategies for treating osteolysis induced by particles are urgently needed. Considering that SIRT1 is a crucial regulator of bone homeostasis and functions as a protective factor against metal nanoparticle-induced inflammatory responses and osteolysis both in vitro and in vivo, SIRT1 may represent a potential therapeutic target in the treatment of aseptic loosening. Another advantage of targeting SIRT1 is that generally adverse effects might not be observed, as most studies have shown positive effects not only on disease treatments but also on lifespan and delayed senescence. ${ }^{58-61}$ Moreover, apart from SIRT1 activators such as resveratrol, SIRT1 can be activated by starvation and calorie restriction, which means that energy metabolism may also play an important role in the treatment of metal nanoparticle-induced osteolysis. However, considering that multiple cell types are involved in the pathological process of aseptic loosening and that SIRT1 has different functions in different cell types and specific conditions, a systematic exploration of the role of SIRT1 in wear PIO and subsequent aseptic loosening is required.

\section{Acknowledgments}

First, we thank all the patients and family members who participated in our study. This study was supported by the National Natural Science Foundation of China (81572111), the Clinical Science and Technology Project Foundation of Jiangsu Province (BL2012002), the Scientific Research Project of Nanjing (201402007), and the Natural Science Foundation of Jiangsu Province (BK20161385).

\section{Disclosure}

The authors report no conflicts of interest in this work.

\section{References}

1. Prokopovich P. Interactions between mammalian cells and nano- or micro-sized wear particles: physico-chemical views against biological approaches. Adv Colloid Interface Sci. 2014;213:36-47.

2. Deng Z, Wang Z, Jin J, et al. SIRT1 protects osteoblasts against particleinduced inflammatory responses and apoptosis in aseptic prosthesis loosening. Acta Biomater. 2017;49:541-554.

3. Purdue PE, Koulouvaris P, Potter HG, Nestor BJ, Sculco TP. The cellular and molecular biology of periprosthetic osteolysis. Clin Orthop Relat Res. 2007;454:251-261.

4. Wang R, Wang Z, Ma Y, et al. Particle-induced osteolysis mediated by endoplasmic reticulum stress in prosthesis loosening. Biomaterials. 2013;34(11):2611-2623.

5. Gallo J, Raska M, Mrazek F, Petrek M. Bone remodeling, particle disease and individual susceptibility to periprosthetic osteolysis. Physiol Res. 2008;57(3):339-349.

6. Kaufman AM, Alabre CI, Rubash HE, Shanbhag AS. Human macrophage response to UHMWPE, TiAlV, $\mathrm{CoCr}$, and alumina particles: analysis of multiple cytokines using protein arrays. J Biomed Mater Res A. 2008;84(2):464-474.

7. Murray DW, Rushton N. Macrophages stimulate bone resorption when they phagocytose particles. J Bone Joint Surg Br. 1990;72(6): 988-992.

8. Kim K, Kobayashi Y, Itoh T. Osteolysis model with continuous infusion of polyethylene particles. Clin Orthop Relat Res. 1998;(352):46-52.

9. Shanbhag AS, Macaulay W, Stefanovic-Racic M, Rubash HE. Nitric oxide release by macrophages in response to particulate wear debris. J Biomed Mater Res. 1998;41(3):497-503.

10. Lei D, Rui W, Zhu YA, et al. Antisense oligonucleotide targeting TNF- $\alpha$ can suppress Co-Cr-Mo particle-induced osteolysis. J Orthop Res. 2008;26(8):1114-1120.

11. Wooley PH, Schwarz EM. Aseptic loosening. Gene Ther. 2004;11(4): 402-407.

12. Pearl JI, Ma T, Irani AR, et al. Role of the Toll-like receptor pathway in the recognition of orthopedic implant wear-debris particles. Biomaterials. 2011;32(24):5535-5542.

13. Hao HN, Zheng B, Nasser S, et al. The roles of monocytic heat shock protein 60 and Toll-like receptors in the regional inflammation response to wear debris particles. J Biomed Mater Res A. 2010;92(4):1373-1381.

14. Hallab NJ, Jacobs JJ. Biologic effects of implant debris. Bull NYU Hosp Jt Dis. 2009;67(2):182-188.

15. Gambhir S, Vyas D, Hollis M, Aekka A, Vyas A. Nuclear factor kappa B role in inflammation associated gastrointestinal malignancies. World $J$ Gastroenterol. 2015;21(11):3174-3183. 
16. Baldwin AS Jr. The NF-kappa B and I kappa B proteins: new discoveries and insights. Annu Rev Immunol. 1996;14:649-683.

17. Dong J, Jimi E, Zeiss C, Hayden MS, Ghosh S. Constitutively active NFkappaB triggers systemic TNFalpha-dependent inflammation and localized TNFalpha-independent inflammatory disease. Genes Dev. 2010; 24(16):1709-1717.

18. Kauppinen A, Suuronen T, Ojala J, Kai K, Salminen A. Antagonistic crosstalk between NF- $\mathrm{KB}$ and SIRT1 in the regulation of inflammation and metabolic disorders. Cell Signal. 2013;25(10):1939-1948.

19. Yeung F, Hoberg JE, Ramsey CS, et al. Modulation of NF-kappaBdependent transcription and cell survival by the SIRT1 deacetylase. EMBO J. 2004;23(12):2369-2380.

20. Rai E, Sharma S, Kaul S, et al. The interactive effect of SIRT1 promoter region polymorphism on type 2 diabetes susceptibility in the North Indian population. PLoS One. 2012;7(11):e48621.

21. Ai TW, Munehiro K, Keizo K, Daisuke K. SIRT1 inactivation induces inflammation through the dysregulation of autophagy in human THP-1 cells. Biochem Biophys Res Commun. 2012;427(1):191-196.

22. Yang H, Zhang W, Pan H, et al. SIRT1 activators suppress inflammatory responses through promotion of $\mathrm{p} 65$ deacetylation and inhibition of NF-kB activity. PLoS One. 2012;7(9):e46364.

23. Rajendrasozhan S, Yang SR, Kinnula VL, Rahman I. SIRT1, an antiinflammatory and antiaging protein, is decreased in lungs of patients with chronic obstructive pulmonary disease. Am J Respir Crit Care Med.2008; 177(8):861-870.

24. Yin H, Ming H, Liang X, et al. Deletion of SIRT1 from hepatocytes in mice disrupts lipin-1 signaling and aggravates alcoholic fatty liver. Gastroenterology. 2014;146(3):801-811.

25. Maya E, Kotas MC, Gillum MP. Sirtuin-1 is a nutrient-dependent modulator of inflammation. Adipocyte. 2013;2(2):113-118.

26. Kwon HS, Brent MM, Getachew R, et al. Human immunodeficiency virus type 1 Tat protein inhibits the SIRT1 deacetylase and induces T-cell hyperactivation. Cell Host Microbe. 2008;3(3):158-167.

27. Borra MT, Smith BC, Denu JM. Mechanism of human SIRT1 activation by resveratrol. J Biol Chem. 2005;280(17):17187-17195.

28. Chen J, Zhou Y, Mueller-Steiner S, et al. SIRT1 protects against microglia-dependent amyloid-beta toxicity through inhibiting NFkappa B signaling. J Biol Chem. 2005;280(48):40364-40374.

29. Sundfeldt M, Carlsson LV, Johansson CB, Thomsen P, Gretzer C. Aseptic loosening, not only a question of wear: a review of different theories. Acta Orthop. 2006;77(2):177-197.

30. Cherian JJ, Jauregui JJ, Samik B, Todd P, Mont MA. What host factors affect aseptic loosening after THA and TKA? Clin Orthop Relat Res. 2015;473(8):2700-2709.

31. Hoenders CSM, Harmsen MC, Luyn MJ. The local inflammatory environment and microorganisms in "aseptic" loosening of hip prostheses. J Biomed Mater Res B Appl Biomater. 2008;86B(86): 291-301.

32. Shen Z, Crotti TN, Mchugh KP, et al. The role played by cell-substrate interactions in the pathogenesis of osteoclast-mediated peri-implant osteolysis. Arthritis Res Ther. 2006;8(3):382-382.

33. Burgess TL, Qian Y, Kaufman S, et al. The ligand for osteoprotegerin (OPGL) directly activates mature osteoclasts. J Cell Biol. 1999;145(3): $527-538$.

34. O’Neill SC, Queally JM, Devitt BM, Doran PP, O’Byrne JM. The role of osteoblasts in peri-prosthetic osteolysis. Bone Joint J. 2013; 95-B(8):1022-1026.

35. Takei H, Pioletti DP, Kwon SY, Sung KLP. Combined effect of titanium particles and TNF- $\alpha$ on the production of IL- 6 by osteoblast-like cells. J Biomed Mater Res. 2000;52(2):382-387.

36. Fujii J, Niida S, Yasunaga Y, Yamasaki A, Ochi M. Wear debris stimulates bone-resorbing factor expression in the fibroblasts and osteoblasts. Hip Int. 2011;21(2):231-237.

37. Wang Z, Huang Z, Gan J, et al. The fibroblast expression of RANKL in CoCrMo-particle-induced osteolysis is mediated by ER stress and XBP1s. Acta Biomater. 2015;24:352-360.
38. Lin TH, Tamaki Y, Pajarinen J, et al. Chronic inflammation in biomaterialinduced periprosthetic osteolysis: NF- $\mathrm{\kappa B}$ as a therapeutic target. Acta Biomater. 2014;10(1):1-10.

39. Wang Z, Liu N, Shi T, et al. ER stress mediates TiAl6V4 particleinduced peri-implant osteolysis by promoting RANKL expression in fibroblasts. PLoS One. 2015;10(9):e0137774.

40. Einav CK, Hanna A, Avi L, et al. Sirt1 is a regulator of bone mass and a repressor of Sost encoding for sclerostin, a bone formation inhibitor. Endocrinology. 2011;152(12):4514-4524.

41. Salvatore F, Giuseppe M, Giovambattista P. Sirt1: def-eating senescence? Cell Cycle. 2012;11(22):4135-4146.

42. Pei-Chi T, Sheng-Mou H, Ruey-Jien C, et al. Resveratrol promotes osteogenesis of human mesenchymal stem cells by upregulating RUNX2 gene expression via the SIRT1/FOXO3A axis. $J$ Bone Miner Res. 2011;26(10):2552-2563.

43. Mehdi S, Constanze B, Ali M. Resveratrol-mediated SIRT-1 interactions with p300 modulate receptor activator of NF-kappaB ligand (RANKL) activation of NF-kappaB signaling and inhibit osteoclastogenesis in bone-derived cells. J Biol Chem. 2011;286(13):11492-11505.

44. Edwards JR, Perrien DS, Nicole F, et al. Silent information regulator (Sir) $\mathrm{T} 1$ inhibits NF- $\mathrm{KB}$ signaling to maintain normal skeletal remodeling. J Bone Miner Res. 2013;28(4):960-969.

45. Huang W, Shang WL, Wang HD, Wu WW, Hou SX. Sirt1 overexpression protects murine osteoblasts against TNF- $\alpha$-induced injury in vitro by suppressing the NF-KB signaling pathway. Acta Pharmacol Sin. 2012; 33(5):668-674.

46. Goodman SB, Huie P, Song Y, et al. Cellular profile and cytokine production at prosthetic interfaces. Study of tissues retrieved from revised hip and knee replacements. J Bone Joint Surg Br. 1998;80(3):531-539.

47. Rae $\mathrm{T}$. The biological response to titanium and titanium-aluminiumvanadium alloy particles. II. Long-term animal studies. Biomaterials. 1986;7(1):30-36.

48. Gülçin İ, Büyükokuroğlu ME, Oktay M, Küfrevioğlu Öİ. Antioxidant and analgesic activities of turpentine of Pinus nigra Arn. subsp. pallsiana (Lamb.) Holmboe. J Ethnopharmacol. 2003;86(1): $51-58$.

49. Inki K, Wenjie X, Reed JC. Cell death and endoplasmic reticulum stress: disease relevance and therapeutic opportunities. Nat Rev Drug Discov. 2009;7(12):1013-1030.

50. Xu C, Bailly-Maitre B, Reed JC. Endoplasmic reticulum stress: cell life and death decisions. J Clin Invest. 2005;115(10):2656-2664.

51. Frand AR, Cuozzo JW, Kaiser CA. Pathways for protein disulphide bond formation. Trends Cell Biol. 2000;10(5):203-210.

52. Rong G, Liu W, Liu B, Zhang B, Li W, Xu Y. SIRT1 suppresses cardiomyocyte apoptosis in diabetic cardiomyopathy: an insight into endoplasmic reticulum stress response mechanism. Int J Cardiol. 2015; 191:36-45.

53. Melhem H, Hansmannel F, Bressenot A, et al. Methyl-deficient diet promotes colitis and SIRT1-mediated endoplasmic reticulum stress. Gut. 2016;65(4):595-606.

54. Lou Y, Wang Z, Xu Y, et al. Resveratrol prevents doxorubicin-induced cardiotoxicity in $\mathrm{H} 9 \mathrm{c} 2$ cells through the inhibition of endoplasmic reticulum stress and the activation of the Sirt1 pathway. Int J Mol Med. 2015;36(3):119-140.

55. Lee J, Hong SW, Park SE, et al. Exendin-4 attenuates endoplasmic reticulum stress through a SIRT1-dependent mechanism. Cell Stress Chaperones. 2014;19(5):649-656.

56. Li Y, Xu S, Giles A, et al. Hepatic overexpression of SIRT1 in mice attenuates endoplasmic reticulum stress and insulin resistance in the liver. FASEB J. 2011;25(5):1664-1679.

57. Goodman SB, Gibon E, Pajarinen J, et al. Novel biological strategies for treatment of wear particle-induced periprosthetic osteolysis of orthopaedic implants for joint replacement. J R Soc Interface. 2014;11(93): 20130962.

58. Michan S, Sinclair D. Sirtuins in mammals: insights into their biological function. Biochem J. 2007;404(1):1-13. 
59. Greer EL, Brunet A. FOXO transcription factors at the interface between longevity and tumor suppression. Oncogene. 2005;24(50): 7410-7425.

60. Finkel T, Deng CX, Mostoslavsky R. Recent progress in the biology and physiology of Sirtuins. Nature. 2009;460(7255):587-591.
61. Bordone L, Guarente L. Calorie restriction, SIRT1 and metabolism: understanding longevity. Nat Rev Mol Cell Biol. 2005;6(4):298-305. 


\section{Supplementary materials}

A
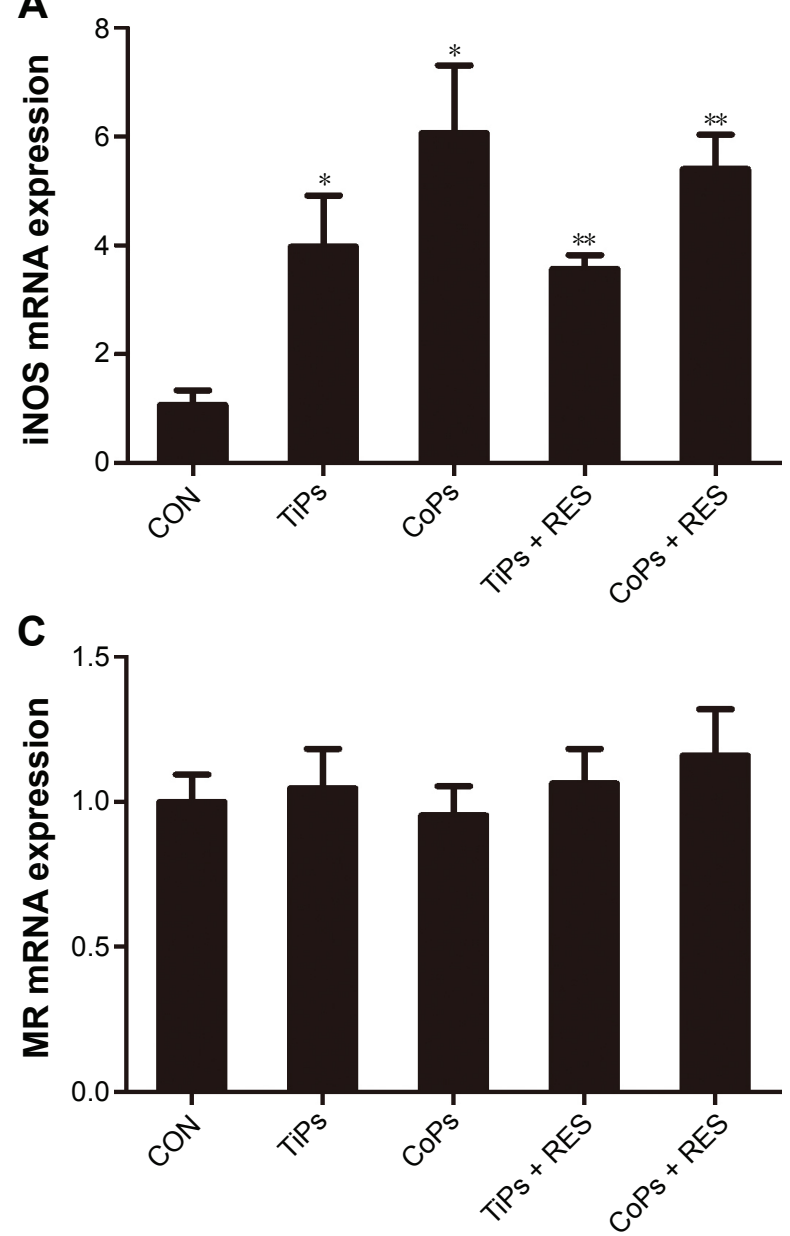

B

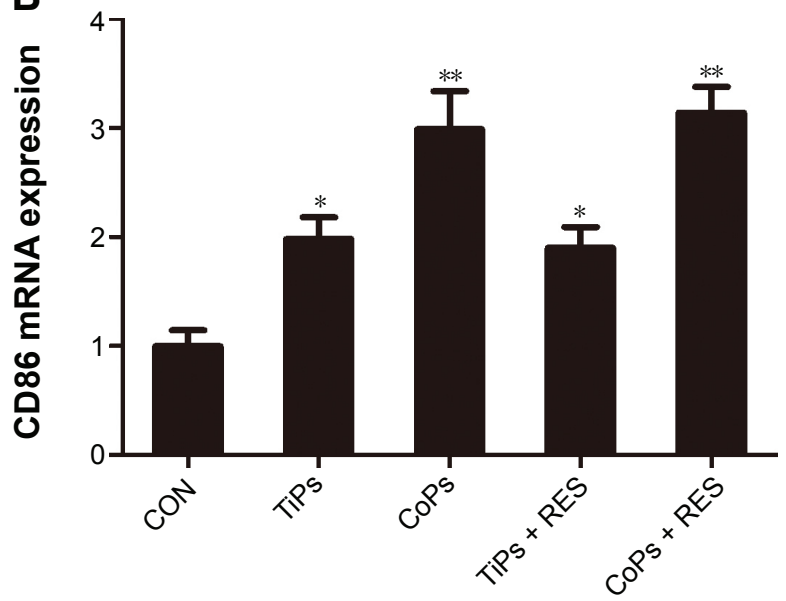

D

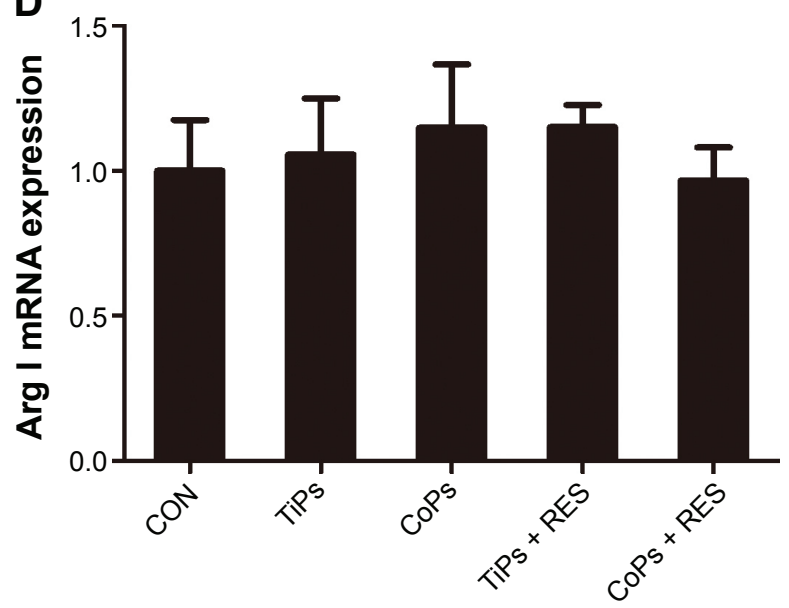

Figure SI Metal nanoparticles induce Raw264.7 cells to differentiate into MI macrophages.

Notes: (A, B) The mRNA expression of iNOS and CD86, which were used as the biomarkers of MI macrophages, was detected by qPCR after the Raw264.7 cells were treated with PBS (control), TiPs, CoPs, TiPs + resveratrol, and CoPs + resveratrol. $* P<0.05, * * P<0.01$ versus CON. (C, D) The mRNA expression of MR and Arg I, which were used as the biomarkers of M2 macrophages, was detected by qPCR after the Raw264.7 cells were treated with PBS (control), TiPs, CoPs, TiPs + resveratrol, and CoPs + resveratrol. The data of all the experiments are represented as the mean \pm SEM from three independent experiments.

Abbreviations: mRNA, messenger RNA; qPCR, quantitative polymerase chain reaction; TiPs, TiAl6V4 particles; CoPs, CoCrMo particles; MR, mannose receptor; SEM, standard error of the mean; RES, resveratrol; CON, control. 


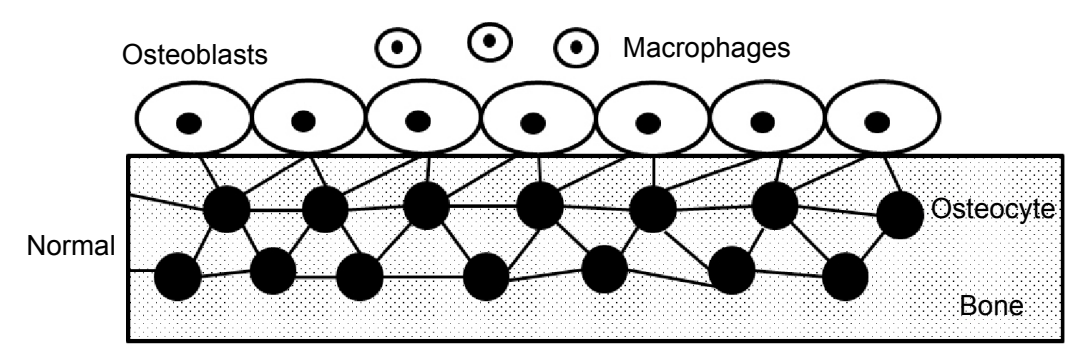

Metal nanoparticles Activated macrophages
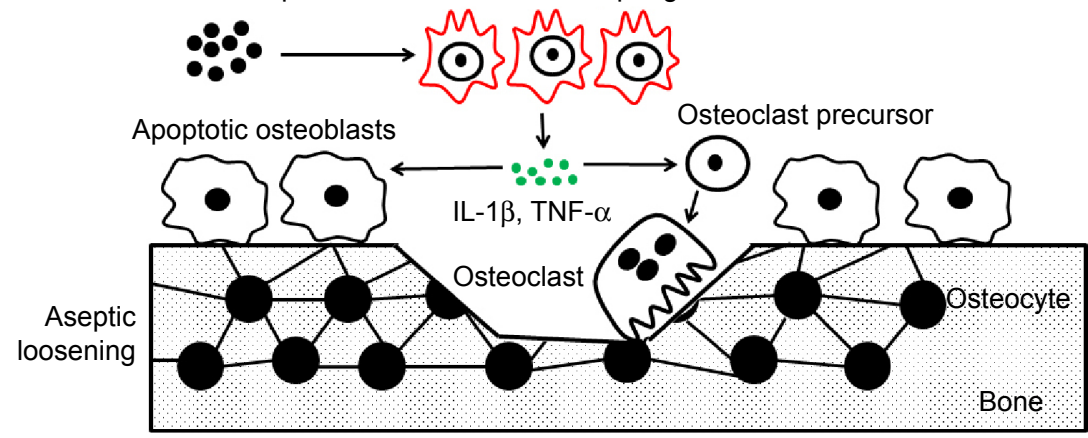

Figure S2 Schematic illustration of the effects of metal nanoparticles on the pathogenesis of aseptic loosening. Notes: It was demonstrated that metal nanoparticle stimulation resulted in the secretion of inflammatory cytokines and further osteoblast apoptosis and osteoclast activation. The imbalance between bone formation and bone resorption eventually resulted in osteolysis. Abbreviations: IL, interleukin; TNF, tumor necrosis factor.

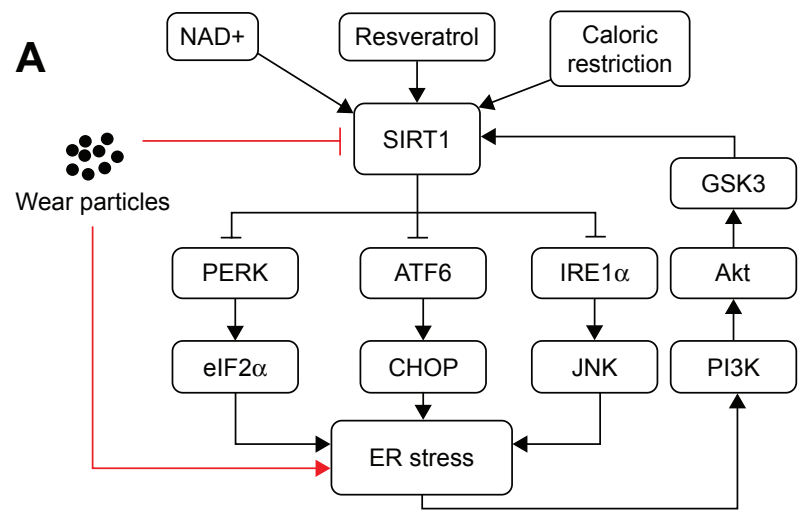

B
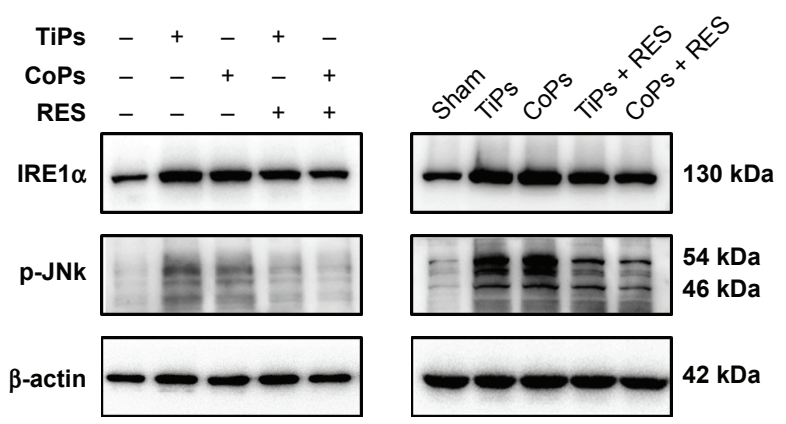

Figure S3 The relationship between ER stress and SIRTI in aseptic loosening.

Notes: (A) Schematic illustration of the signaling network between SIRTI and ER stress. (B) Western blots performed after Raw264.7 cells were treated with PBS (control), TiPs, CoPs, TiPs + resveratrol, and CoPs + resveratrol.

Abbreviations: ER, endoplasmic reticulum; SIRTI, Sirtuin I; PBS, phosphate-buffered saline; TiPs, TiAI6V4 particles; CoPs, CoCrMo particles; NAD+, nicotinamide adenine dinucleotide; RES, resveratrol. 


\section{Publish your work in this journal}

The International Journal of Nanomedicine is an international, peerreviewed journal focusing on the application of nanotechnology in diagnostics, therapeutics, and drug delivery systems throughout the biomedical field. This journal is indexed on PubMed Central, MedLine, CAS, SciSearch $\AA$, Current Contents $\AA /$ Clinical Medicine,

Journal Citation Reports/Science Edition, EMBase, Scopus and the Elsevier Bibliographic databases. The manuscript management system is completely online and includes a very quick and fair peer-review system, which is all easy to use. Visit http://www.dovepress.com/ testimonials.php to read real quotes from published authors.

Submit your manuscript here: http://www.dovepress.com/international-journal-of-nanomedicine-journal 